\title{
Operational macroscopic modeling of complex urban road intersections
}

\author{
Gunnar Flötteröd ${ }^{*} \quad$ Jannis Rohde ${ }^{\dagger}$
}

December 4, 2010

Report TRANSP-OR 101204

Transport and Mobility Laboratory

Ecole Polytechnique Fédérale de Lausanne

transp-or.epfl.ch

${ }^{*}$ Transport and Mobility Laboratory, Ecole Polytechnique Fédérale de Lausanne, CH-1015 Lausanne, Switzerland, gunnar.floetteroed@epfl.ch (corresponding author)

${ }^{\dagger}$ Institute of Transportation and Urban Engineering, Technische Universität Braunschweig, D38106 Braunschweig, Germany, j.rohde@tu-braunschweig.de 


\begin{abstract}
This article describes a new approach to the macroscopic first order modeling and simulation of traffic flow in complex urban road intersections. The framework is theoretically sound, operational, and comprises a large body of models presented so far in the literature.

Working within the generic node model class of Tampere et al. (forthcoming), the approach is developed in two steps. First, building on the incremental transfer principle of Daganzo et al. (1997), an incremental node model for general road intersections is developed. A limitation of this model (as of the original incremental transfer principle) is that it does not capture situations where the increase of one flow decreases another flow, e.g., due to conflicts. In a second step, the new model is therefore supplemented with the capability to describe such situations. A fixed-point formulation of the enhanced model is given, solution existence and uniqueness are investigated, and two solution algorithms are developed. The feasibility and realism of the new approach is demonstrated through both a synthetic and a real case study.
\end{abstract}

\title{
1 Introduction
}

A traffic flow model describes vehicular dynamics given a certain traffic infrastructure and, if applicable, given additional route choice information. The infrastructure comprises the considered road (system) in terms of geometry, speed limits, and such. If a complete network is given, this also includes intersection properties such as turning move limitations, right-of-way laws, and signaling, and it requires supplementary route choice information. The vehicular dynamics may be described at various levels of details, ranging from single-vehicle interactions ("car-following models", e.g., Brackstone and McDonald, 1999; Pandawi and Dia, 2005), over partially aggregate ("mesoscopic") models (e.g., Astarita et al., 2001; Ben-Akiva et al., 2001; De Palma and Marchal, 2002; Mahmassani, 2001; Nökel and Schmidt, 2002), to fully macroscopic models that treat vehicular traffic flows as continuous streams. The latter model class is considered in this article. The major advantage of macroscopic models is their tractable mathematical structure and their low number of parameters.

Macroscopic models for traffic flow on a link have gone from the fundamental diagram (where density and velocity are uniquely related and flow is a function of either density or velocity (Greenshields, 1935)) via the Lighthill-WhithamRichards theory of kinematic waves (where the fundamental diagram is inserted 
into an equation of continuity (Lighthill and Witham, 1955; Richards, 1956)) to second-order models (where a second equation introduces inertia (Payne, 1971)). This article concentrates on the kinematic wave model (KWM), but it also adds empirical evidence to the ongoing debate if more complex models yield a substantial gain in expressive power (e.g., Lebacque and Lesort, 1999; Hoogendoorn and Bovy, 2001; Nagel and Nelson, 2005).

Instances of the KWM are collectively referred to as "first order models" because they model velocity (first order information) but do not explicitly specify an acceleration/deceleration law (which would constitute second order information). Macroscopic first order models capture a bird's view on traffic flow: They do not distinguish individual vehicles, and they allow arbitrarily abrupt velocity changes; both features are consistent with reality only if sufficiently large space- and timescales are considered. These properties of the KWM apply well to freeway traffic but are more difficult to associate with urban traffic, which is dominated by intersection dynamics. Also, urban intersections can be far more complex than the simple three-legged junctions (merges and diverges) one encounters on freeways. Furthermore, certain urban intersection conflicts can be safely resolved based on right-of-way laws that go without signaling. Hence, the adequate modeling of urban traffic with the KWM requires to represent this type of intersections as well. This is the objective of this article. It is achieved in two steps.

First, the incremental transfer (IT) principle of Daganzo et al. (1997) is developed further into a solution procedure for the generic node model (GNM) of Tampere et al. (forthcoming). The original IT principle simulates multi-lane multi-class traffic flow. Although the problem of modeling the outflow of a set of upstream lanes into a set of downstream lanes bears clear similarities with the general intersection modeling problem, no systematic effort to carry the IT principle over to general intersections has been reported in the literature. Therefore, an incremental node model (INM) is developed that (i) inherits the intuitive appeal and computational convenience of the IT principle, (ii) generalizes the IT principle in that it allows for flow transfer rates that vary continuously during the transmission (iii) is consistent with the GNM, and (iv) comprises a large body of first order intersection models presented so far in the literature.

Second, the disability of both the IT principle and the INM to capture situations where the increase of one flow leads to the decrease of another flow is overcome. This situation occurs mainly when one traffic stream yields to another, and an increase in the high-priority stream decreases the throughput of the low-priority stream. The INM is therefore extended with concrete node supply constraints that capture such interactions. These constraints (i) allow to describe complex flow interactions within the node, (ii) are still consistent with the GNM, and (iii) can be conveniently included in the INM through a fixed point formulation. More 
generally, these node supply constraints apply to a wider model class than the GNM; they are compatible with every node model that is phrased in terms of the KWM's demand/supply framework.

The resulting INM with node supply constraints (INMC) is investigated with respect to solution existence and uniqueness, and two solution procedures are developed. This analysis reveals that (i) flows in point-like intersection models can be non-unique even for simple three-armed topologies and that (ii) problems of this kind are likely to result from parameter misspecifications in combination with the limitations of the point-like modeling approach as such. Furthermore, it is shown that situations exist where any instance of the GNM resolves this ambiguity by selecting a solution that would result from cooperative instead of competitive driver behavior. A modification of the GNM that overcomes this confinement is therefore proposed. Overall, this analysis indicates that intersections beyond a certain spatial complexity should indeed be modeled spatially, at least by distinguishing several point-like intersection elements. However, the improved modeling power of the INMC it is clearly demonstrated through both a synthetic and a real case study.

The remainder of this article is organized as follows. Subsections 1.1 and 1.2 review the demand/supply framework of the KWM and the GNM in order to enable a self-contained presentation. Section 2 develops the INM, puts it in relation with the existing literature, and clarifies its basic working through an example. Section 3 then enriches the INM with node supply constraints, discusses solution existence and uniqueness of the resulting INMC, and develops two solution procedures. Section 4 evaluates the new model both in terms of a synthetic and a real example. Finally, Section 5 concludes the article.

\subsection{Demand/supply framework for the kinematic wave model}

The KWM requires a minimal set of assumptions to model traffic flow on a linear road. Denote by $x \in \mathbb{R}$ a location on that road and by $t \in \mathbb{R}$ the continuous time. $\rho(x, t)$ is the local density (in vehicle units per length unit) of traffic, $q(x, t)$ is its flow (in vehicle units per time unit), and $v(x, t)$ is its velocity. These quantities are related by the first constituent equation of the KWM:

$$
q(x, t)=v(x, t) \rho(x, t) .
$$

The second modeling assumption is that of vehicle conservation. On smooth conditions, it is expressed by the continuity equation

$$
\frac{\partial \rho}{\partial t}+\frac{\partial q}{\partial x}=0
$$




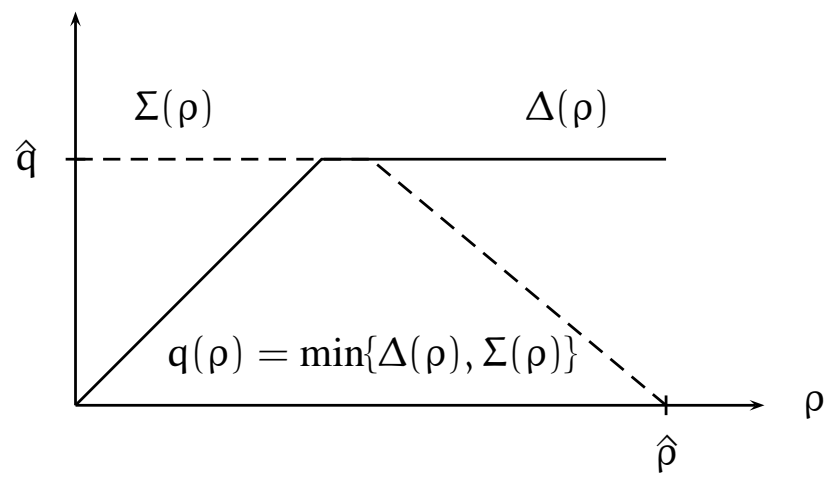

Figure 1: Local demand and supply comprise a fundamental diagram The demand function $\Delta(\rho)$ (solid) consists of an increasing part with its slope equal to the free flow speed and is limited by the flow capacity $\hat{q}$. The supply function $\Sigma(\rho)$ (dashed) is also limited by the flow capacity. The slope of its declining part equals the backward wave speed and intersects the abscissa at the greatest possible density $\hat{\rho}$. The minimum of both functions yields a fundamental diagram. Piecewise linearity is a typical but not necessary property; the demand/supply framework applies to all concave and nondecreasing demand and supply functions.

Finally, local flow is specified as a function of local density only. This relation is usually denoted as the fundamental diagram:

$$
q(x, t)=Q(\rho(x, t), x) .
$$

Since these specifications can still result in ambiguities, an additional condition must be instrumented to select the physically relevant solution. Given a concave fundamental diagram, the principle of local demand and supply provides a convenient technique to ensure uniqueness (Daganzo, 1994, 1995a,b; Lebacque, 1996; Nelson and Kumar, 2006). Denote by $x-(x+)$ the location immediately upstream (downstream) of $x$. For every $x$, the local flow $q(x, t)$ is then defined as the minimum of local flow demand $\Delta(\rho(\mathrm{x}-, \mathrm{t}), \mathrm{x}-)$ and local flow supply $\Sigma(\rho(x+, t), x+)$ :

$$
q(x, t)=\min \{\Delta(\rho(x-, t), x-), \Sigma(\rho(x+, t), x+)\} .
$$

Figure 1 illustrates this function.

Equation (4) reflects the self-evident constraint that local traffic flow is bounded by the flow that can be dismissed from the immediate upstream location and by the 


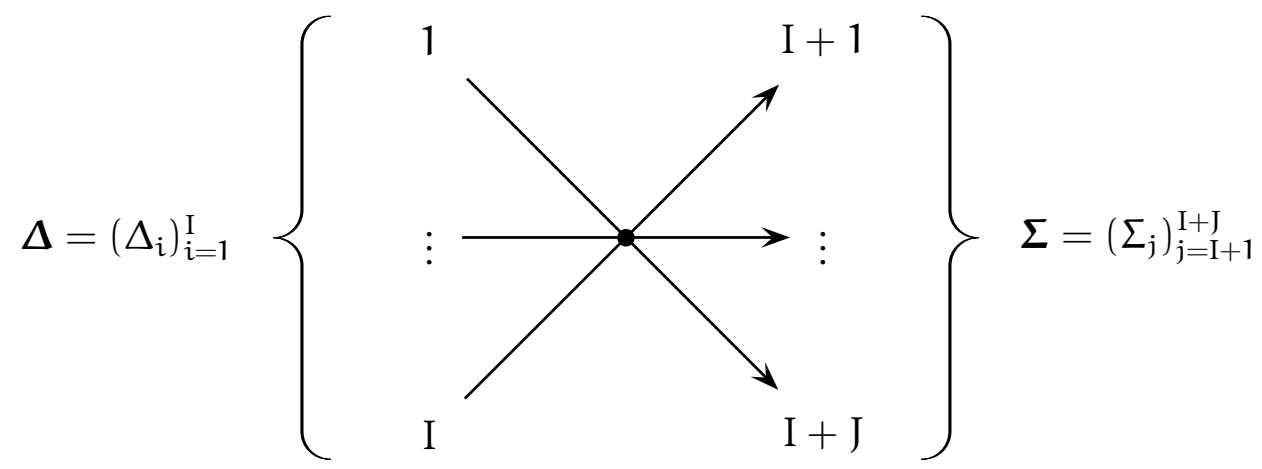

Figure 2: A general intersection with I ingoing and J outgoing links

flow that can be absorbed by the immediately downstream location. Furthermore, the local flow is maximized subject to these constraints. This property enforces the physically relevant solution of the KWM. Phenomenologically, it is a statement of drivers' ride impulse (Ansorge, 1990), which is equivalently expressed by the microsimulation rule for cellular automata "Drive as fast as you can and stop [only] if you have to!" (Chrobok et al., 2003; Daganzo, 2006).

Beyond its ability to uniquely capture traffic flow on a link, this principle also applies to general intersections, cf. Figure 2 . In such a setting, every upstream link $i$ provides a demand $\Delta_{i}(t)$ equal to its greatest possible inflow into the intersection, and every downstream link $j$ provides a supply $\Sigma_{j}(t)$ equal to the greatest possible outflow it accepts from the intersection. Additional phenomenological modeling is facilitated since these boundaries alone are generally not sufficient to uniquely define the intersection flows. However, every reasonable specification must adhere to the principle of local flow maximization subject to all phenomenological constraints.

\subsection{Generic macroscopic node model}

Macroscopic node models that are based on constrained flow maximization aim at selecting the physically relevant solutions of the KWM while facilitating further phenomenological modeling through the constraints. Early models along these lines are described by Holden and Risebro (1995); Coclite et al. (2005); Herty and Klar (2003), who, however, resort to unrealistic assumptions about the turning behavior of drivers. Lebacque and Khoshyaran (2005) define a node model 
class that maximizes a concave function of the flow and comprises the models of Holden and Risebro (1995) and Coclite et al. (2005).

The GNM of Tampere et al. (forthcoming), on which this article builds, follows a similar approach, yet with a simpler objective function and more detailed constraints. A node model belongs to this class if it solves the following optimization problem:

$$
\begin{aligned}
\max _{\mathbf{q}^{\text {in }}} & \sum_{i=1}^{\mathrm{I}} \mathrm{q}_{i}^{\text {in }} \\
\text { s.t. } & \mathbf{q} \geq \mathbf{0} \\
& \mathbf{q}^{\text {out }}=\mathbf{B} \mathbf{q}^{\text {in }} \\
& \mathbf{q}^{\text {in }} \leq \boldsymbol{\Delta} \\
& \mathbf{q}^{\text {out }} \leq \mathbf{\Sigma} \\
& \mathbf{q} \text { satisfies the invariance principle } \\
& \mathbf{q} \text { satisfies a supply constraint interaction rule }
\end{aligned}
$$

where the vector $\mathbf{q}^{\text {in }}=\left(q_{i}^{\text {in }}\right)$ collects the I node inflows from upstream, the vector $\mathbf{q}^{\text {out }}=\left(\mathbf{q}_{j}^{\text {out }}\right)$ collects the J node outflows towards downstream, $\mathbf{q}=\left(\begin{array}{c}\mathbf{q}^{\text {in }} \\ \mathbf{q}^{\text {out }}\end{array}\right)$, $\Delta=\left(\Delta_{i}\right)$ and $\boldsymbol{\Sigma}=\left(\Sigma_{j}\right)$ are vectors of upstream demands and downstream supplies, and $\mathbf{B}=\left(\beta_{i j}\right)$ is a matrix of non-negative turning fractions from upstream arcs $i$ to downstream arcs $j$ with $\sum_{j} \beta_{i j}=1$. There are no dynamics within the node, and its spatial extension is neglected. (Lebacque $(1984,2003)$ offers dynamic node models.) The GNM is defined for a discrete time step or a single point in continuous time; the time index is omitted here for simplicity. The following presentation assumes discrete time due to its focus on operational model implementations.

Equations (5) - (9) have straightforward interpretations: The objective function (5) calls for flow maximization. Equation (6) requires non-negative flows. Vehicle conservation within the node and compliance with the turning fractions (which are specified exogenously here but can be functions of the upstream flow composition) are required in (7). Equations (8) and (9) require that node inflows and outflows do not exceed the respective demands and supplies.

The invariance principle in (10) states the following (Lebacque and Khoshyaran, 2005):

$$
\mathbf{q} \text { is invariant with respect to } \begin{cases}\Delta_{i} \rightarrow C_{i} & \text { for all upstream } i \text { with } q_{i}<\Delta_{i} \\ \Sigma_{j} \rightarrow C_{j} & \text { for all downstream } j \text { with } q_{j}<\Sigma_{j},\end{cases}
$$


where $C_{i}\left(C_{j}\right)$ is the flow capacity of upstream link $i$ (downstream link $j$ ). Timediscrete models that violate the invariance principle allow for waves that emanate from the node while at the same time traveling towards the node; these solutions are unstable when the time step size approaches zero. Intuitively, the invariance principle can be phrased as follows: (i) increasing the queue size on a congested upstream link has no effect on the node flows (because the link flow is constrained from downstream), and (ii) making more space available on an uncongested downstream link does not change the node flows (because the link flow is constrained from upstream).

Equations (11) and (12) facilitate further phenomenological modeling. The supply constraint interaction rule $(S C I R)$ in (11) determines how a limited downstream supply is shared among the upstream links in congested conditions. It is a necessary element of every node model with more than one upstream link. The node supply constraints in (12) capture additional effects that reduce the node flows, in particular signaling and conflicts inside the node. They are optional elements in that it is possible to compute unique node flows even without node supply constraints.

\section{Incremental node model}

This section develops a new node model that is related to the IT principle. This principle was originally formulated as a discrete-time simulation procedure for a freeway with special lanes and priority vehicles (Daganzo et al., 1997); see Lawson et al. (1999) for a qualitatively validation of the underlying model and Zhang and Lin (2001) for a review. The IT principle computes flows per time step by incrementally transferring flow elements from upstream to downstream until some termination criterion is met.

Daganzo et al. (1997) already conjecture a broader applicability of the IT principle but do not develop this consideration further. Indeed, despite of its intuitive appeal, the IT principle has never been systematically investigated as a method to compute traffic flow in a general intersection. ${ }^{1}$ Lebacque and Koshyaran (2002) relate their lane assignment model to the multi-lane model solved by the IT principle, but they turn to different techniques when it comes to node modeling. Laval and Daganzo (2006) deploy a variant of the IT principle when analyzing the effect of lane changing in traffic streams. They observe the similarity of their flow transmission rules with some intersection models of Lebacque and Lesort (1999);

\footnotetext{
${ }^{1}$ This statement is supported by an inspection of all 19 articles that cite Daganzo et al. (1997) up to December 3, 2010 according to the Scopus citation database (Elsevier, accessed 2010).
} 
however, this observation is not further pursued. Also, Laval and Daganzo (2006) deploy a demand-proportional supply distribution logic, which violates the invariance principle. Their lane-changing model is picked up by Cheu et al. (2009), again deploying a demand-proportional supply distribution scheme.

The subsequently developed incremental node model (INM) features consistency with the GNM, simple and intuitive parametrization, and computational efficiency. The latter property is crucial in every non-trivial network model, which may consist of many thousand node instances. Furthermore, the INM truly generalizes all instances of the GNM proposed so far in the literature.

\subsection{Specification}

The incremental transfer of flow occurs along a fictitious time line $t$, with the transfer starting at $t=0$. The flow being transferred until $t$ is written as $\mathbf{q}(t)$, and the finally transmitted flow is $\mathbf{q}=\mathbf{q}(\infty)$. The INM is specified in terms of a stationary point of the following dynamical system:

$$
\begin{aligned}
\dot{\mathbf{q}}(\mathrm{t}) & =\boldsymbol{\varphi}(\mathbf{q}(\mathrm{t})) \\
\text { s.t. } \mathbf{q}(0) & =\mathbf{0} \\
\boldsymbol{\varphi}(\cdot) & \geq \mathbf{0}
\end{aligned}
$$

The differential equation (14) represents the incremental transfers, which start at zero flows according to (15). The requirement (16) of non-negative flow transfer rates $\varphi(\cdot)$ ensures that flows do not become negative, which satisfies (6). In the following, a set of (increasingly stringent) constraints on $\varphi(\cdot)$ that ensure full consistency with the GNM is developed.

The constraint (7) is equivalent to

$$
\varphi^{\text {out }}(\mathbf{q})=\mathbf{B} \varphi^{\text {in }}(\mathbf{q})
$$

with $\varphi(\cdot)=\left(\begin{array}{c}\varphi^{\text {in }}(\cdot) \\ \varphi^{\text {out }}(\cdot)\end{array}\right)$. Equation (17) results from (7) by differentiation with respect to $t$, and it uniquely implies (7) by integration because of (15).

To enforce the demand and supply constraints (8) and (9), the set

$$
D(\mathbf{q})=\left\{l \mid \begin{array}{ll}
1 \leq l \leq I \text { (upstream): } & q_{l}^{\text {in }}<\Delta_{l} \text { and }\left\{j \mid \beta_{l j}>0\right\} \subseteq D(\mathbf{q}) \\
I<l \leq I+J(\text { downstream): } & q_{l}^{\text {out }}<\Sigma_{l} \text { and } \exists i \in D(\mathbf{q}): \beta_{i l}>0
\end{array}\right\}
$$

is defined. It contains those upstream (downstream) arcs that are not demand (supply) constrained. Furthermore,

$$
\varphi_{\mathrm{l}}(\mathbf{q})=\mathbf{1}(l \in \mathrm{D}(\mathbf{q})) \cdot \phi_{\mathrm{l}}(\mathbf{q})
$$


where $\mathbf{1}(\cdot)$ is the indicator function and

$$
\boldsymbol{\phi}(\mathbf{q})=\left(\phi_{\mathrm{l}}(\mathbf{q})\right)=\left(\begin{array}{c}
\boldsymbol{\phi}^{\mathrm{in}}(\mathbf{q}) \\
\boldsymbol{\phi}^{\text {out }}(\mathbf{q})
\end{array}\right)=\left(\begin{array}{c}
\mathbf{I} \\
\mathbf{B}
\end{array}\right) \boldsymbol{\phi}^{\text {in }}(\mathbf{q})>\mathbf{0}
$$

with $\mathbf{I}$ being the $I \times I$ identity matrix. This definition still fulfills (17). The strict positivity of $\boldsymbol{\phi ( \cdot )}$ in combination with (18) and (19) guarantees that the transfer process eventually attains a stationary point, at which $\mathrm{D}=\{\}$ must hold. Equations (18)-(20) implicitly define the SCIRs that can be expressed with the INM through an appropriate choice of $\boldsymbol{\phi}^{\text {in }}(\cdot)$.

The INM is by design consistent with the invariance principle: At every solution of the model, $\mathrm{D}$ is the empty set. Consider a solution $\mathrm{q}_{i}^{\text {in }}<\Delta_{i}$. The first part of (18) implies that there is a downstream link $j$ with $\beta_{i j}>0$ and $q_{j}^{\text {out }}=\Sigma_{j}$ that keeps $i$ from sending more flow downstream. Increasing $\Delta_{i}$ does not change this situation, and hence the first part of (13) is satisfied. For a solution $q_{j}^{\text {out }}<\Sigma_{j}$, the second part of (18) implies that there is no upstream link $i$ that could send more flow towards $j$. Increasing $\Sigma_{j}$ does not change this situation, and hence the second part of (13) is satisfied.

Finally, the stationary point $\mathbf{q}(\infty)$ of the INM is flow maximizing: Every feasible setting of $\Delta, \Sigma$, and $\phi^{\text {in }}(\cdot)$ defines a unique incremental transfer path $\{\mathbf{q}(t)\}_{t=0}^{\infty}$, which obeys the SCIR constraint. The objective function (5) increases monotonously along this path because of

$$
\left\langle\frac{\partial\left(\sum_{i} q_{i}^{\text {in }}\right)}{\partial \mathbf{q}}, \boldsymbol{\varphi}(\mathbf{q})\right\rangle=\sum_{i} \varphi_{i}^{\text {in }}(\mathbf{q}) \geq 0 .
$$

$\mathrm{D}(\mathbf{q}(\infty))=\{\}$ implies that all elements of $\mathbf{q}(\infty)$ are either demand- or supply-constrained. Demand-constrained inflows cannot be increased. Supplyconstrained inflows can only be increased by reducing at least one competing inflow, say $i$. Denote by $\tilde{q}_{i}^{\text {in }}<q_{i}^{\text {in }}(\infty)$ the accordingly reduced inflow. The monotonicity of the SCIR path that led to $\mathbf{q}(\infty)$ guarantees the existence of a transfer time $\tilde{\mathrm{t}}$ with $\mathrm{q}_{i}^{\text {in }}(\tilde{\mathrm{t}})=\tilde{\mathrm{q}}_{i}^{\text {in }}$, cf. (15) and (16). However, $\mathrm{q}_{i}^{\text {in }}(\tilde{\mathrm{t}})<\mathrm{q}_{i}^{\text {in }}(\infty)$ implies $\varphi_{i}^{\text {in }}(\mathbf{q}(\tilde{\mathfrak{t}}))>0$ and hence $\mathrm{q}_{i}^{\text {in }}(\tilde{\mathrm{t}})<\tilde{\mathrm{q}}_{i}^{\text {in }}$, which is a contradiction. This means that any reduction of an inflow component in $\mathbf{q}(\infty)$ is incompatible with the SCIR. Hence, $\mathbf{q}(\infty)$ is uniquely flow maximizing subject to the phenomenological constraints.

\subsection{Instances of the incremental node model}

To specify a concrete instance of the INM, only $\boldsymbol{\phi}^{\text {in }}(\cdot)>\mathbf{0}$ needs to be defined. A general approach is (i) to specify a parametric $\phi^{\text {in }}(\cdot ; \boldsymbol{\alpha})$ that captures the structure 
of the considered intersection and (ii) to calibrate the parameter vector $\alpha$ from data. An appealing choice is a piecewise constant $\phi^{\text {in }}(\cdot ; \boldsymbol{\alpha})$ : In terms of calibration, this enables an intuitive procedure of incrementally adding thresholds to $\alpha$ until a sufficient data fit is obtained. To give an example, Troutbeck and Kako (1999) indicate that low-priority vehicles merge more aggressively at high flows. This can be modeled by assigning a relatively low $\phi^{\text {in }}$ to the respective stream for low $q$ values and using a larger $\phi^{\text {in }}$ if $q$ exceeds an appropriate threshold. The important computational advantage of this approach is that piecewise constant transfers allow to replace the integration of (14) by a finite sum.

The most extreme case of this specification is $\boldsymbol{\phi}^{\text {in }}(\cdot ; \boldsymbol{\alpha}) \equiv \boldsymbol{\alpha}=\left(\alpha_{i}\right)$, which assigns a constant inflow priority $\alpha_{i}$ to every upstream arc such that the ratios of node inflows in perfectly over-saturated conditions equal the ratios of their $\alpha$ coefficients. To the best of the authors' knowledge, all instances of the GNM presented so far in the literature can be phrased as an INM with $\phi^{\text {in }}(\cdot ; \boldsymbol{\alpha}) \equiv \boldsymbol{\alpha}$ and a particular choice of $\alpha$. These models are enumerated in the following; the according "proofs" of equivalence follow from a comparison of the respective simulation procedures with the basic INM solution algorithm given in the next subsection.

Not surprisingly, the first-in/first-out (FIFO) diverge logic of the INM coincides with many other FIFO diverges, including those of Daganzo (1995a), Lebacque (1996), Adamo et al. (1999) $)^{2}$, Kuwahara and Akamatsu (2001) $)^{3}$, Lebacque and Koshyaran (2002), Jin and Zhang (2004), Gentile et al. (2007), Nie et al. (2008), Yperman et al. (2007), Bliemer (2007), and Nie et al. (2008) .

The merge model of $\mathrm{Ni}$ and Leonard (2005) results from the INM with a single downstream link and letting all $\alpha_{i}$ equal to the respective upstream link flow capacities. The merge model of Daganzo (1995a) coincides with the INM for two upstream links and one downstream link as long as all inflow priorities are strictly positive. Kuwahara and Akamatsu (2001) given an incomplete specification of a full merge model that could be made consistent with the INM, which would essentially result in a model of the $\mathrm{Ni}$ and Leonard (2005) type.

The unsignalized intersection model of Tampere et al. (forthcoming) generalizes the merge model of $\mathrm{Ni}$ and Leonard (2005). It also results from the INM by setting

\footnotetext{
${ }^{2}$ Adamo et al. (1999) specify a FIFO condition in their Eq. (20), but their solution (23) does not exhibit this property. Their experimental results only consider a merge, hence it can only be speculated that the model is indeed FIFO.

${ }^{3}$ The specification of Kuwahara and Akamatsu (2001) is redundant in that the second constraint in their Eq. (16) never binds.

${ }^{4}$ The "max" operator in (19) of Nie et al. (2008) should actually be a "min" that creates a FIFO diverge logic (Nie, 2010).
} 
the $\alpha_{i}$ parameters equal to the upstream link flow capacities. Similarly, the signalized intersection model of Tampere et al. (forthcoming) can be captured by scaling the respective $\alpha_{i}$ parameters with the green time fractions of their upstream links.

Many discrete-time merge and general node models in the literature are based on a demand-proportional distribution of the downstream supplies. All of these models violate the invariance principle (Lebacque and Khoshyaran, 2005) and are hence incompatible with both the GNM and the INM. This applies to the models of Buisson et al. (1995); Buisson, Lebacque and Lesort (1996); Buisson, Lebacque, Lesort and Mongeot (1996), Rubio-Ardanaz et al. (2001), Jin and Zhang (2003), Jin and Zhang (2004), Bliemer (2007), Yperman et al. (2007), van Hinsbergen et al. (2008), Nie et al. (2008), and Kurzhanskiy and Varaiya (2010).

The general intersection models of Gentile et al. (2007) and Adamo et al. (1999) as well as the merge model of Lebacque (1996) are not flow maximizing; the latter also allows, without further correction, for solutions where the flow exceeds the supply. This also excludes them from the GNM and INM framework.

\subsection{Basic implementation of the incremental node model}

In the following, a solution procedure for the most basic instance of the INM with a constant $\phi^{\mathrm{in}}(\cdot)$ is described. This specification results in piecewise constant flow transfer rates $\varphi(\cdot)$, where changes occur when a new demand or supply constraint become active, cf. (19). The flow transmissions are hence computed in a multistage process with stage index $k=0 \ldots K$. The flows at the beginning of stage $k$ are denoted by $\mathbf{q}^{(\mathrm{k})}=\left(\begin{array}{c}\mathbf{q}^{\text {in }(\mathrm{k})} \\ \mathbf{q}^{\text {out }(\mathrm{k})}\end{array}\right)$ and

$$
\mathbf{q}^{(0)}=\mathbf{0}
$$

is let consistently with (15).

The parameter $\theta^{(k)}$ defines the length of stage $k$ on the fictitious time line of the incremental transfer process, such that

$$
\mathbf{q}^{(\mathrm{k}+1)}=\mathbf{q}^{(\mathrm{k})}+\theta^{(\mathrm{k})} \boldsymbol{\varphi}\left(\mathbf{q}^{(\mathrm{k})}\right)
$$

where $\boldsymbol{\varphi}\left(\mathbf{q}^{(\mathrm{k})}\right)$ is defined through (18) and (19). Since stage $k$ must end when the first flow in $\mathrm{D}^{(\mathrm{k})}=\mathrm{D}\left(\mathbf{q}^{(\mathrm{k})}\right)$ reaches a constraint,

$$
\theta^{(k)}=\min _{l \in D^{(k)}}\left\{\begin{array}{ll}
\left(\Delta_{l}-q_{l}^{\text {in }(k)}\right) / \varphi_{l}^{\text {in }}\left(\mathbf{q}^{(k)}\right) & \text { for l upstream } \\
\left(\Sigma_{l}-q_{l}^{\text {out }(k)}\right) / \varphi_{l}^{\text {out }}\left(\mathbf{q}^{(k)}\right) & \text { for l downstream }
\end{array}\right\} .
$$




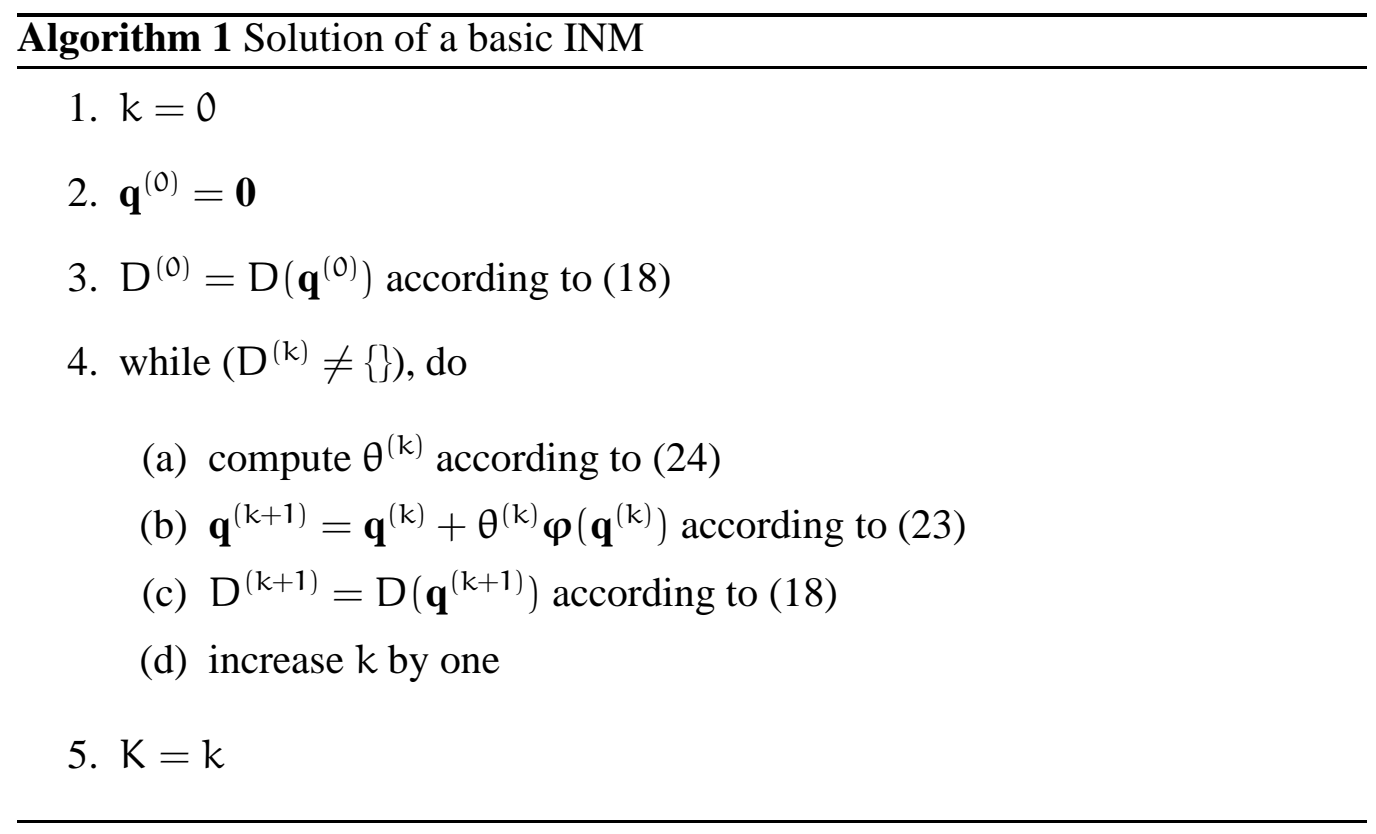

The overall process is summarized in Algorithm 1. It terminates in stage $\mathrm{K}$ where $\mathrm{D}^{(\mathrm{K})}=\{\}$ and, equivalently, $\boldsymbol{\varphi}^{(\mathrm{K})}=\mathbf{0}$. This algorithm solves only the most basic INM instance with a constant $\phi^{\text {in }}$ function. The general INM with a variable $\boldsymbol{\phi}^{\text {in }}$ function can be solved by numerical integration of (14)-(16). The INM with a piecewise constant $\phi^{\text {in }}$ function is solved by a straightforward generalization of Algorithm 1.

This algorithm reproduces all of the aforementioned FIFO diverge models, the merge model of Daganzo (1995a), the merge model of Ni and Leonard (2005), and the unsignalized intersection model of (Tampere et al., forthcoming). This can be shown by walking through the computation steps described in these references and comparing them to Algorithm 1, which establishes that all of these models are instances of the basic INM with a constant $\phi^{\text {in }}$ function. The specification given here is very compact and comes without the lengthy proofs of Tampere et al. (forthcoming) because its consistency with the GNM results immediately from the fact that it is an instance of the INM. It should also be noted that the basic Algorithm 1 was first heuristically proposed by Flötteröd and Nagel (2005) and later shown to be flow-maximizing by Flötteröd (2008). The next subsection clarifies its working through an example.

In summary, this subsection demonstrates that, to the best of the authors' knowledge, all instances of the GNM presented so far in the literature are covered by the basic INM. Beyond this, the presented framework renders the future specification of much richer GNM instances through the INM with a variable $\phi^{\text {in }}$ function a 
technically straightforward exercise. However, the remaining presentation will be in terms of the basic INM with a constant $\phi^{\text {in }}$ function only.

\subsection{Example}

The considered test network is shown in Figure 3. It will be revisited in later parts of this article, where the INM is compared to a detailed traffic microsimulator. This section only demonstrates the workings of the basic INM as specified in Algorithm 1.

The network consists of a major street in north/south direction, which is intersected by a minor one way street that runs from east to west. There are three upstream (predecessor) links $\mathrm{P}_{\mathrm{S}}, \mathrm{P}_{\mathrm{E}}, \mathrm{P}_{\mathrm{N}}$ and three downstream (successor) links $S_{N}, S_{W}, S_{S}$. Traffic coming from the south enters the intersection from predecessor link $P_{S}$, traffic coming from the east enters it via $P_{E}$, and traffic coming from the north enters it via $P_{N}$. The links $S_{N}, S_{W}$, and $S_{S}$ represent the northbound, westbound, and southbound exit of the intersection, respectively. 50 percent of the traffic coming from $P_{S}$ turns left at the intersection, and 50 percent of the traffic coming from $P_{N}$ turns right at the intersection, both into successor link $S_{W}$. The traffic on the minor street only crosses the intersection. Summarizing, $\beta_{\mathrm{SN}}=0.5$, $\beta_{\mathrm{SW}}=0.5, \beta_{\mathrm{EW}}=1, \beta_{\mathrm{NW}}=0.5$, and $\beta_{\mathrm{NS}}=0.5$. The right-of-way laws at the westbound merge $S_{\mathrm{W}}$ are modeled through the priority values $\alpha_{\mathrm{N}}=10, \alpha_{\mathrm{S}}=1$, and $\alpha_{\mathrm{E}}=0.1$.The further parameters of this intersection are described later in Section 4.1, where they become relevant for the first time.

Consider first an uncongested scenario, where the downstream supplies are sufficient to absorb all upstream demands. In particular, the following demand and supply values are assumed: $\Delta_{\mathrm{S}}=600 \mathrm{veh} / \mathrm{h}, \Delta_{\mathrm{E}}=100 \mathrm{veh} / \mathrm{h}, \Delta_{\mathrm{N}}=600 \mathrm{veh} / \mathrm{h}$, $\Sigma_{\mathrm{N}}=1400 \mathrm{veh} / \mathrm{h}, \Sigma_{\mathrm{W}}=1400 \mathrm{veh} / \mathrm{h}$, and $\Sigma_{\mathrm{S}}=1400 \mathrm{veh} / \mathrm{h}$. Table 1 details the flow transmissions in these conditions. Every block of rows in this table corresponds to one stage of the INM. All upstream demands are fully served: The simulated flow rates are $q_{S}^{\text {in }}=600 \mathrm{veh} / \mathrm{h}, q_{E}^{\text {in }}=100 \mathrm{veh} / \mathrm{h}, \mathrm{q}_{\mathrm{N}}^{\text {in }}=600 \mathrm{veh} / \mathrm{h}$, $\mathrm{q}_{\mathrm{N}}^{\text {out }}=300 \mathrm{veh} / \mathrm{h}, \mathrm{q}_{\mathrm{W}}^{\text {out }}=700 \mathrm{veh} / \mathrm{h}$, and $\mathrm{q}_{\mathrm{S}}^{\text {out }}=300 \mathrm{veh} / \mathrm{h}$. All node inflows are split proportionally to their respective turning fractions.

The following setting represents a congested scenario: $\Delta_{\mathrm{S}}=600 \mathrm{veh} / \mathrm{h}, \Delta_{\mathrm{E}}=$ $100 \mathrm{veh} / \mathrm{h}, \Delta_{\mathrm{N}}=600 \mathrm{veh} / \mathrm{h}, \Sigma_{\mathrm{N}}=1400 \mathrm{veh} / \mathrm{h}, \Sigma_{\mathrm{W}}=400 \mathrm{veh} / \mathrm{h}$, and $\Sigma_{\mathrm{S}}=$ $1400 \mathrm{veh} / \mathrm{h}$. The total demand for $S_{\mathrm{W}}$ is $\beta_{\mathrm{SW}} \Delta_{\mathrm{S}}+\beta_{\mathrm{EW}} \Delta_{\mathrm{E}}+\beta_{\mathrm{NW}} \Delta_{\mathrm{N}}=700 \mathrm{veh} / \mathrm{h}$, which exceeds its supply of $400 \mathrm{veh} / \mathrm{h}$. Table 2 details how this situation is simulated in the INM. In stage 0 , no constraint binds, all node inflows are increased proportionally to their priorities, and all node outflows are increased by inflows that result from weighting the upstream inflows with the turning fractions. The 


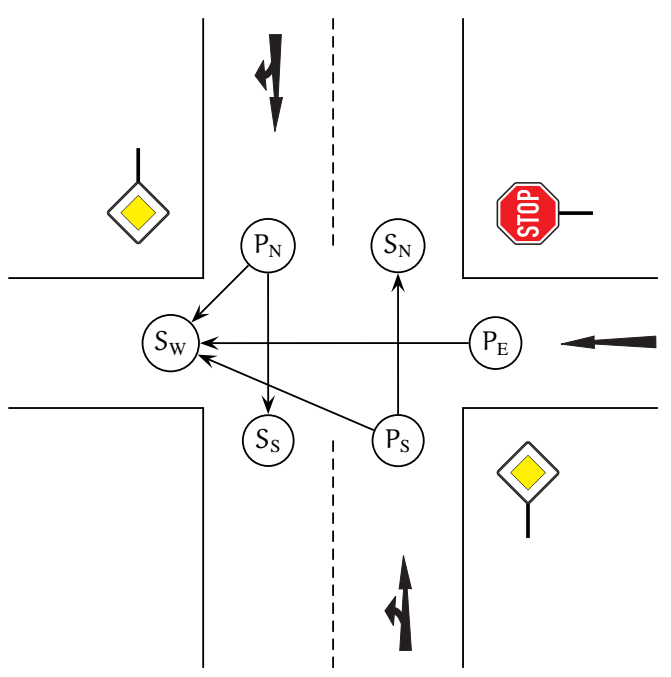

Figure 3: Test network

See Section 2.4 for a detailed description.

Table 1: Uncongested flow transmissions

\begin{tabular}{|l|c|c|c|c|c|c|}
\hline & $\mathrm{P}_{\mathrm{S}}$ & $\mathrm{P}_{\mathrm{E}}$ & $\mathrm{P}_{\mathrm{N}}$ & $\mathrm{S}_{\mathrm{N}}$ & $\mathrm{S}_{\mathrm{W}}$ & $\mathrm{S}_{\mathrm{S}}$ \\
\hline \hline $\mathrm{q}^{(0)}$, cf. $(15)$ & 0 & 0 & 0 & 0 & 0 & 0 \\
\hline$\varphi^{(0)}$, cf. $(19)$ & 1 & 0.1 & 10 & 0.5 & 5.6 & 5 \\
\hline$\left(\Delta / \Sigma-\mathrm{q}^{(0)}\right) / \varphi^{(0)}$ & 600 & 1000 & 60 & 2800 & 250 & 280 \\
\hline$\theta^{(0)}, \mathrm{cf}(24)$ & \multicolumn{6}{|c|}{60} \\
\hline \hline $\mathrm{q}^{(1)}, \mathrm{cf} .(23)$ & 60 & 6 & 600 & 30 & 336 & 300 \\
\hline$\varphi^{(1)}$ & 1 & 0.1 & 0 & 0.5 & 0.6 & 0 \\
\hline$\left(\Delta / \Sigma-\mathrm{q}^{(1)}\right) / \varphi^{(1)}$ & 540 & 940 & $\%$ & 2740 & 1773.33 & $\infty$ \\
\hline$\theta^{(1)}$ & \multicolumn{7}{|c|}{540} \\
\hline \hline $\mathrm{q}^{(2)}$ & 600 & 60 & 600 & 300 & 660 & 300 \\
\hline$\varphi^{(2)}$ & 0 & 0.1 & 0 & 0 & 0.1 & 0 \\
\hline$\left(\Delta / \Sigma-\mathrm{q}^{(2)}\right) / \varphi^{(2)}$ & $\%$ & 400 & $\%$ & $\infty$ & 7400 & $\infty$ \\
\hline$\theta^{(2)}$ & 600 & 100 & 600 & 300 & 700 & 300 \\
\hline \hline $\mathrm{q}^{(3)}$ & 0 & 0 & 0 & 0 & 0 & 0 \\
\hline$\varphi^{(3)}$ & 600 &
\end{tabular}

Every block of rows represents one flow transmission stage. The algorithm terminates when all flow transmissions have ceased. The simulated flow rates are given in the second last row. ( $\Delta / \Sigma$ is to be read as "either $\Delta$ or $\Sigma$ ".) 
Table 2: Congested flow transmissions

\begin{tabular}{|l|c|c|c|c|c|c|}
\hline & $\mathrm{P}_{\mathrm{S}}$ & $\mathrm{P}_{\mathrm{E}}$ & $\mathrm{P}_{\mathrm{N}}$ & $\mathrm{S}_{\mathrm{N}}$ & $\mathrm{S}_{\mathrm{W}}$ & $\mathrm{S}_{\mathrm{S}}$ \\
\hline \hline $\mathrm{q}^{(0)}$ & 0 & 0 & 0 & 0 & 0 & 0 \\
\hline$\varphi^{(0)}$ & 1 & 0.1 & 10 & 0.5 & 5.6 & 5 \\
\hline$\left(\Delta / \Sigma-\mathrm{q}^{(0)}\right) / \varphi^{(0)}$ & 600 & 1000 & 60 & 2800 & 71.43 & 280 \\
\hline$\theta^{(0)}$ & \multicolumn{7}{|c|}{60} \\
\hline \hline $\mathrm{q}^{(1)}$ & 60 & 6 & 600 & 30 & 336 & 300 \\
\hline$\varphi^{(1)}$ & 1 & 0.1 & 0 & 0.5 & 0.6 & 0 \\
\hline$\left(\Delta / \Sigma-\mathrm{q}^{(1)}\right) / \varphi^{(1)}$ & 540 & 940 & $\%$ & 2740 & 106.67 & $\infty$ \\
\hline$\theta^{(1)}$ & \multicolumn{7}{|c|}{106.67} \\
\hline \hline $\mathrm{q}^{(2)}$ & 166.67 & 16.67 & 600 & 83.34 & 400 & 300 \\
\hline$\varphi^{(2)}$ & 0 & 0 & 0 & 0 & 0 & 0 \\
\hline
\end{tabular}

Every block of rows represents one flow transmission stage. The algorithm terminates when all flow transmissions have ceased. The simulated flow rates are given in the second last row.

inflow from $\mathrm{P}_{\mathrm{N}}$, which has the highest priority and hence the highest transfer rate, reaches its demand constraint most quickly. Next, the downstream supply of $S_{W}$ is used up in stage 1. Since all upstream links have a non-zero turning fraction towards $S_{\mathrm{W}}$, all flow transmissions cease in stage 2 such that the algorithm terminates. The simulated flow rates are $q_{S}^{\text {in }}=166.67 \mathrm{veh} / \mathrm{h}, q_{\mathrm{E}}^{\text {in }}=16.67 \mathrm{veh} / \mathrm{h}$, $\mathrm{q}_{\mathrm{N}}^{\text {in }}=600 \mathrm{veh} / \mathrm{h}, \mathrm{q}_{\mathrm{N}}^{\text {out }}=83.34 \mathrm{veh} / \mathrm{h}, \mathrm{q}_{\mathrm{W}}^{\text {out }}=400 \mathrm{veh} / \mathrm{h}$, and $\mathrm{q}_{\mathrm{S}}^{\text {out }}=300 \mathrm{veh} / \mathrm{h}$. The node inflow from $\mathrm{P}_{\mathrm{N}}$ is completely transmitted because its priority is high enough to reserve sufficient capacity in $S_{\mathrm{W}}$. The remainder of $S_{\mathrm{W}}$ 's capacity is completely used up by $P_{S}$ and $P_{E}$, the flows of which are proportional to their priorities.

These experiments demonstrate how the basic INM simulates a complex intersection. However, this ability also introduces new difficulties: Conflicting streams (in this example: the left- turning portion of the northbound flow vs. the southbound flow and the minor flow vs. all major flows) are not yet accounted for. The following section shows how they can be consistently incorporated in the model.

\section{Node supply constraints}

The GNM's optional node supply constraints have so far been neglected. They capture node capacity reductions that result from signaling and/or conflicts inside the node. See, e.g, Rouphail et al. (1999) for capacity rules in signalized intersec- 
tions and Troutbeck and Brilon (1999) for unsignalized intersections. The INM is now supplemented with generic node supply constraints through an additional fixed-point condition. This combines the computational efficiency of the INM with vastly increased modeling capabilities, and it also yields some interesting insights into the workings of the GNM. However, the proposed representation of node supply constraints is not limited to the GNM but applies more generally to any node model that is based on the KWM's demand/supply framework.

Node supply constraints complicate the modeling substantially. Consider, for example, the case of an unsignalized intersection with conflicting streams of different priorities. If a stream yields, the FIFO diverge logic reduces all node inflows from its upstream arc. This makes more space available in the downstream merges, which in turn may allow other flows to increase. These flows may be of sufficiently high priority such that again other flows may have to yield to them. These dependencies are complex and, possibly, even circular.

Subsection 3.1 adds node supply constraints to the INM through a fixed point formulation. Subsection 3.2 discusses specification and uniqueness issues. Finally, Subsection 3.3 presents two solution algorithms of different scope and complexity.

\subsection{Specification}

The INM (14) - (20) is formally written as

$$
\mathbf{q}=\left(\begin{array}{c}
\mathbf{q}^{\text {in }} \\
\mathbf{q}^{\text {out }}
\end{array}\right)=\operatorname{INM}(\Delta, \Sigma),
$$

where the parameters $\alpha$ of the underlying $\phi$ function are omitted for notational simplicity. (Algorithm 1 could be used to solve this model for a constant $\boldsymbol{\phi}$ function; however, a more complex specification with a variable $\boldsymbol{\phi}$ function is just as feasible.)

The node supply constraints are implemented through a demand constraint function $\widehat{\Delta}(\mathbf{q})$ that bounds the original demand $\Delta$, given a flow pattern $\mathbf{q}$ in the intersection. The suchlike constrained demands $\Delta(\mathbf{q})$ result from

$$
\Delta(\mathbf{q})=\min \{\Delta, \widehat{\Delta}(\mathbf{q})\}
$$

where the min function applies element by element. The use of a demand constraint function is no modeling confinement: Even though node supply constraints may apply to individual traffic streams, the streams from each upstream arc are 
uniquely coupled through the FIFO rule, which allows to combine their node supply constraints into a single demand constraint function. ${ }^{5}$

The INM with node supply constraints (INMC) thus requires to identify flow rates $\mathbf{q}$ that solve the fixed-point problem

$$
\begin{aligned}
\mathbf{q} & =\operatorname{INM}(\Delta(\mathbf{q}), \boldsymbol{\Sigma}) \\
& =\operatorname{INM}(\min \{\Delta, \widehat{\Delta}(\mathbf{q})\}, \boldsymbol{\Sigma}) .
\end{aligned}
$$

The equivalence of this equation with a GNM where the node supply constraints (12) are phrased as $\mathbf{q}^{\text {in }} \leq \boldsymbol{\Delta}(\mathbf{q})$ strongly depends on the concrete specification of the node model. For example, since (27) constitutes a necessary condition for the GNM with node supply constraints, the existence and uniqueness of a solution of (27) would also guarantee a unique solution of the GNM. However, the following analysis shows that full consistency with the GNM is a delicate issue.

A solution of (27) is guaranteed to exist if the demand constraint function $\widehat{\Delta}(\mathbf{q})$ is continuous and applies to every $\mathbf{q}$ between $\mathbf{0}$ and $\left(\begin{array}{c}\Delta \\ \Sigma\end{array}\right)$ : The basic INM constitutes a continuous mapping of demands on flows because it results from an integration of the flow transfer rates $\varphi(\cdot)$. For a continuous demand constraint function $\widehat{\Delta}(\mathbf{q})$, the combined function $\operatorname{INM}(\min \{\boldsymbol{\Delta}, \widehat{\Delta}(\mathbf{q})\}, \boldsymbol{\Sigma})$ is therefore continuous with respect to $\mathbf{q}$ as well. This function maps every flow $\mathbf{q}$ from the closed, bounded, and convex set $\mathbf{0} \leq \mathbf{q} \leq\left(\begin{array}{c}\Delta \\ \boldsymbol{\Sigma}\end{array}\right)$ on this very set. That is, the model (27) specifies a fixed point of a continuous mapping of a closed, bounded, and convex set on itself. Brouwer's fixed point theorem guarantees that this mapping has at least one fixed point (Sobolev, 2001). Subsection 3.2 is devoted to the less obvious uniqueness analysis of (27).

The modeling of node supply constraints through demand constraint functions has been tentatively pursued by some authors. van Hinsbergen et al. (2008, 2009) capture stream line interactions within an intersection by constraining the respective demand functions dependently on the demand for conflicting flows of higher priority. That is, they replace the demand constraint function $\widehat{\Delta}(\mathbf{q})$ by a constraint function $\widehat{\Delta}(\boldsymbol{\Delta})$ that does not depend on the actual flows $q$ in the intersection but on the demands $\Delta$. There are simple situations where this approach fails. Assume, for example, an upstream demand $\Delta_{1}$ that affects some other upstream demand

\footnotetext{
${ }^{5}$ For example, assume that every stream from upstream link $i$ to downstream link $j$ is faced with an individual node supply constraint, which is expressed in terms of a stream-specific demand constraint function $\widehat{\Delta}_{i j}(\mathbf{q})$. These constraints can be combined through the FIFO rule into one joint demand constraint function $\widehat{\Delta}_{i}(\mathbf{q})=\min _{j}\left\{\widehat{\Delta}_{i j}(\mathbf{q}) / \beta_{i j}\right\}$.
} 
$\Delta_{2}$ via $\widehat{\Delta}_{2}\left(\Delta_{1}\right)$. Now, consider any situation where the actually transmitted portion of $\Delta_{1}$ becomes arbitrarily small, e.g., because of a congested downstream arc or because of an interaction with another traffic stream. In the most extreme case, nothing of $\Delta_{1}$ is transmitted at all such that its impact on the intersection effectively vanishes - but $\widehat{\Delta}_{2}\left(\Delta_{1}\right)$ invariably creates the same effect as if all of $\Delta_{1}$ actually entered the intersection. Lebacque (1984) models flow-dependent demand constraints but solves the model linearly in that mutual dependencies are not resolved. Yperman et al. (2007) also identify the need for truly flow-dependent demand constraints, but they neither specify nor solve such a model.

\subsection{Uniqueness analysis}

By incorporating node supply constraints in the INM, two models are connected: The basic INM is designed to identify the unique solution of a subclass of the GNM. The demand constraint function $\widehat{\Delta}(\mathbf{q})$ should be defined in a unique manner as well. However, without further assumptions there is no guarantee that the combined model (27) also has a unique solution. In this section, it is demonstrated that non-uniqueness is likely to result from misspecifications that are intimately related to the limitations of point-like intersection models as such. It also is shown that the flow maximizing approach of the GNM should be revised because it selects counter-intuitive results in certain situations where the fixed-point model allows for multiple solutions.

Consider the situation shown in Figure 4. This is a three-armed intersection, where a fraction $\beta_{\mathrm{NW}}$ of flow $q_{\mathrm{N}}^{\text {in }}$, coming from the north, turns into the westbound link $\mathrm{W}$ and the remainder continues southbound. A fraction $\beta_{S W}$ of flow $q_{S}^{\text {in }}$, coming from the south, also turns into link $\mathrm{W}$, while the remainder continues northbound. The left-turning share of $q_{S}^{\text {in }}$ yields to the southbound share of $q_{N}^{\text {in }}$. Traffic is single-lane in every direction. This is a simplified version of the previously used test network, where the minor stream coming from the east is removed for greatest clarity.

To begin with, the yielding of the left-turning flow is ignored and it is assumed that the flow supply $\Sigma_{\mathrm{W}}$ of the westbound link is the limiting factor of the intersection's throughput and that neither the demand $\Delta_{\mathrm{N}}$ nor the demand $\Delta_{\mathrm{S}}$ can be fully served such that

$$
\beta_{\mathrm{NW}} q_{\mathrm{N}}^{\text {in }}+\beta_{\mathrm{SW}} q_{\mathrm{S}}^{\text {in }}=q_{\mathrm{W}}^{\text {out }}=\Sigma_{\mathrm{W}} .
$$

This relation is represented by line 1 in Figure 5. Given the priorities $\alpha_{N}$ and $\alpha_{S}$ for the two ingoing streams,

$$
\frac{\beta_{\mathrm{SW}} q_{\mathrm{S}}^{\text {in }}}{\beta_{\mathrm{NW}} q_{\mathrm{N}}^{\text {in }}}=\frac{\alpha_{\mathrm{S}}}{\alpha_{\mathrm{N}}}
$$




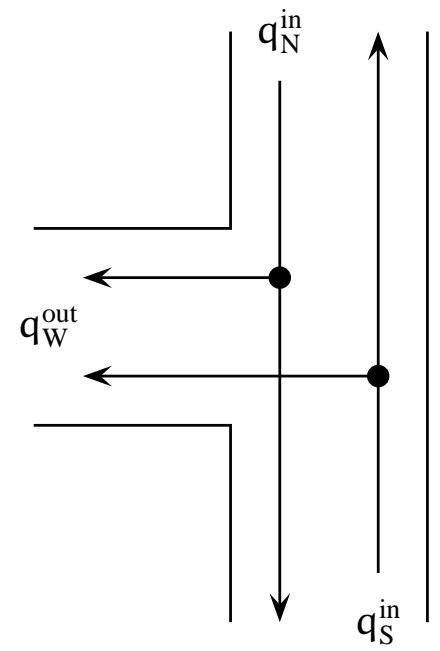

Figure 4: Simplified test network

A three-armed intersection, where some of flow $\mathrm{q}_{\mathrm{N}}^{\text {out }}$, coming from the north, turns into the westbound link and the remainder continues southbound. A portion of flow $q_{S}^{\text {out }}$, coming from the south, also turns into the westbound link, while the remainder continues northbound. The left-turning share of $q_{S}^{\text {out }}$ yields to the southbound share of $q_{N}^{\text {out }}$. Traffic is single-lane in every direction.

holds furthermore in these conditions. An exemplary instance of this relation is given by line 2. Its intersection with line 1 at point $\mathrm{A}$ constitutes the unique solution of the unconstrained node model.

Now, let the left-turning portion of $\mathrm{q}_{\mathrm{S}}^{\text {in }}$ yield to the straight portion of $\mathrm{q}_{\mathrm{N}}^{\text {in }}$. The according demand constraint function $\widehat{\Delta}_{S}\left(q_{N}^{\text {in }}\right)$ is represented by curve 3 in Figure 5 . A feasible solution point must not be located above this curve. Point A maintains to be a feasible solution of the constrained model because the new constraint is not violated. However, an inspection of point B now reveals the following properties: (i) given the transmitted flows, the constraint binds but is not violated, and (ii) an evaluation of the INM with an accordingly constrained demand reproduces the flows.

That is, both A and B are fixed points of (27), and hence both solve this intersection model. The cause of this ambiguity is the priority specification: Line 2 has a very large slope of $\alpha_{S} / \alpha_{N}$, which implies that $q_{S}^{\text {in }}$ precedes $q_{N}^{\text {in }}$ in the westbound merge. On the other hand, the node supply constraint indicates the opposite: The left-turning portion of $\mathrm{q}_{\mathrm{S}}^{\text {in }}$ yields to the southbound part of $\mathrm{q}_{\mathrm{N}}^{\text {in }}$. If the priorities were selected such that line 2 was flat enough to intersect curve 3 before reaching line 1, then point B would be the unique solution. Furthermore, if the slope 


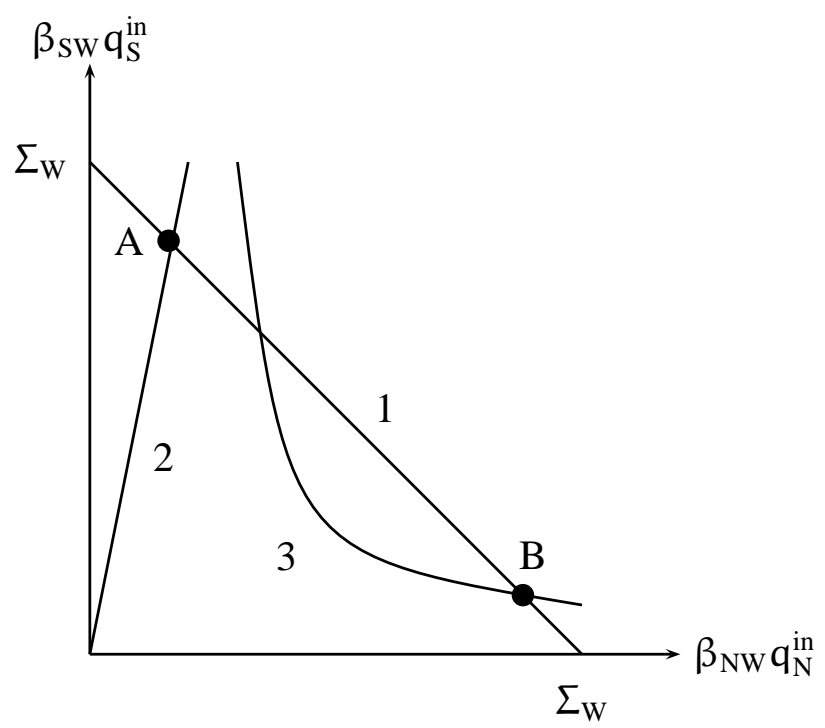

Figure 5: Ambiguous intersection flows

Given this constellation of the merge constraint 1 , the priority constraint 2 , and the endogenous flow constraint 3, there are two possible flow solutions A and B for the intersection of Figure 4.

of line 2 was so low that it intersected line 1 below point $\mathrm{B}$, then only this new intersection point would solve the model.

The non-uniqueness in this example clearly results from a misspecified model. This type of misspecification is not easy to identify automatically, which is demonstrated by a slight modification of the setting. Consider the modified version of the previous example shown in Figure 6. The only difference is that $q_{N}^{\text {in }}$ is now allowed to u-turn and merge into the northbound part of $q_{S}^{\text {in }}$. A realistic priority rule for this setting would be that the the u-turning stream yields to the straight stream. This can lead to a situation where the node supply constraint on the left-turning portion of $q_{S}^{\text {in }}$ becomes inactive because the u-turning stream is held back such that it blocks the $q_{N}^{\text {in }}$ stream, which otherwise would constrain $q_{S}^{\text {in }}$. This situation yields a flow diagram that looks qualitatively identical to Figure 5, only that line 1 now represents the merge constraint of the northern downstream arc and line 2 represents the priorities at this merge. In this case, point A represents indeed a plausible solution.

Unfortunately, returning from the fixed point model (27) to the original GNM does not help to resolve this ambiguity. A combination of (28) with (29) shows that point $A$ is globally flow maximizing if $\beta_{\mathrm{NW}}<\beta_{\mathrm{SW}}$, point $\mathrm{B}$ is globally flow maximizing if $\beta_{\mathrm{NW}}>\beta_{\mathrm{SW}}$, and both points yield the same global flows if $\beta_{\mathrm{NW}}=\beta_{\mathrm{SW}}$. However, there is no reason why a change in the turning fractions 


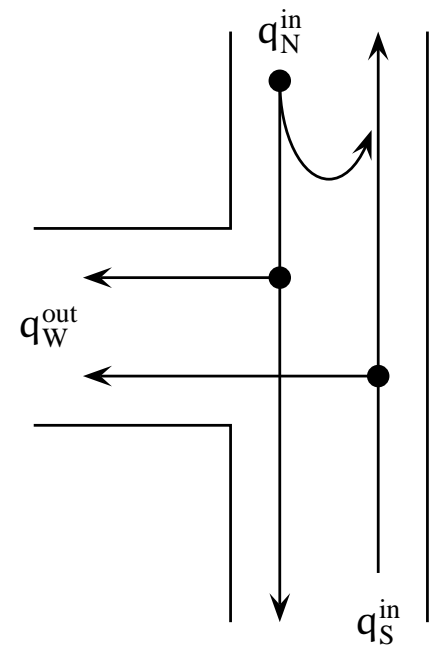

Figure 6: Simplified test network with additional u-turn

The only difference to the intersection of Figure 4 is that the traffic coming from the north is now allowed to u-turn and merge into the straight northbound stream.

should favor one solution over the other, and hence the maximization of flows can only be attributed to some kind of cooperative driver behavior across different streams.

This cooperative behavior results from the GNM's maximization of one joint objective function (5). An arguably more realistic approach is to phrase the node model as an I-player game where each inflow is one player, the continuous-valued strategy set of a player is the amount of flow the player sends into the intersection, and the individual-level objective function to be maximized by each player is the amount of flow sent into the node. This would result in a truly competitive intersection model the flows of which would be in a Nash equilibrium.

The model (27) can be seen as a fixed-point formulation of such an equilibrium. This means that it is indeed behaviorally plausible that the fixed point model is ambiguous where the GNM is not. This raises the question of the GNM's scope. The authors consider the GNM appropriate in situations where the INM is applicable as well, because solution uniqueness can be guaranteed. However, the introduction of node supply constraints seems to reach the limits of the original GNM. A fixed-point reformulation of the GNM appears to be a logical next step in future research.

Considering the wealth of thinkable intersection models, the generality of the INMC reaches the limitations of what situations can be reasonably modeled by point-like intersections. If, for a very complex intersection, the spatial arrange- 


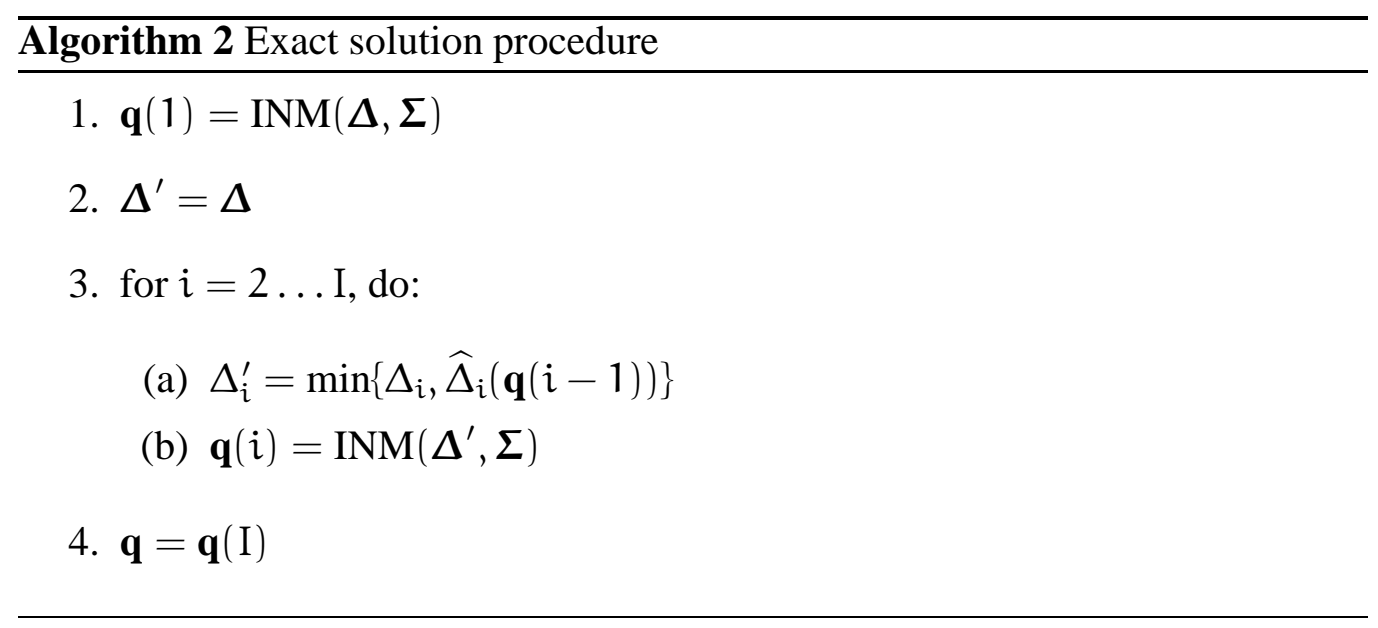

ment of interactions contains information that is necessary for a unique model specification, then this intersection should indeed be modeled spatially, e.g., by linking several point-like intersection components. However, the question if welldesigned real intersections permit systematically ambiguous flow patterns is yet to be answered. It may be that the problems observed here are consequences of pathological specifications that would not be considered by an experienced analyst. Still, all of these critical statements does not invalidate the observation that the INMC advances the macroscopic first order modeling of complex intersections considerably.

\subsection{Solution algorithms}

In the following, two solution methods for the fixed point problem (27) are described. Both approaches terminate after a finite number of calculations and are experimentally tested in the next section. The first method yields an exact solution but requires an independence assumption to be satisfied. The second method is universally applicable but may yield only an approximate solution. For notational simplicity, it is assumed that every node inflow $i=1 \ldots$ I is subject to a demand constraint function that represents one or more node supply constraints.

Exact solution procedure. Assume that the upstream arcs $i=1 \ldots$ I can be ordered such that the node inflow from $i$ is independent of all node inflows $i+$ 1 ... I in terms of both the INM and the node supply constraints. (This ordering is not unique if there are mutually independent inflows.) Then, (27) can be solved in I steps by Algorithm 2. 
First, the basic INM is run without demand constraints, which yields the flows $\mathbf{q}(1)$. Based on this, the second highest ranking demand $i=2$ is reduced according to the demand constraint function $\widehat{\triangle}_{2}(\mathbf{q}(1))$. By assumption, this constraint does not change in reaction to variations in the node inflows from $i=3 \ldots$. Then, the basic INM is run again, using the constrained demand of $i=2$, which yields the flows $\mathbf{q}(2)$. Because of the independence assumption, the constraint on $i=2$ binds exactly in this and all following iterations. Now, the demand of $i=3$ is constrained by $\widehat{\triangle}_{3}(\mathbf{q}(2))$, the INM is run again, and so forth. After I steps, all constraints are satisfied, and the simulated flows reproduce these constraints.

Approximate solution procedure. If no ranking of the node inflows is possible because of mutual dependencies, Algorithm 3 can be deployed. It yields an approximate solution of (27) with only two evaluations of the INM, between which it interpolates both the flows and the constraints. This procedure can also be deployed as a building block in a more precise iterative solution algorithm, which, however, is beyond the scope of this article.

The steps 1 and 2 identify the two working points A and B between which the flows and the constraints are interpolated. Step 1 calculates the unconstrained flows A and the constraints A that result from these flows. Step 2 then calculates the flows B given the constraints A and identifies the new constraints B that result from the flows B. Step 3 solves the intersection model based on linearly interpolated flows and constraints. In particular, step 3a identifies for every upstream link $i$ the interpolation coefficient $\lambda_{i}^{*}$ at which $i$ 's interpolated constraint binds. If a constraint bind at either working point, flow $\mathrm{A}$, which effectively ignores the constraints, is assumed, which corresponds to $\lambda_{i}^{*}=1$. Otherwise, $\lambda_{i}^{*}$ results from equating the linearized flow and its constraint, solving for $\lambda_{i}^{*}$, and ensuring that no extrapolation takes place by projecting $\lambda$ on the interval $[0,1]$. Step $3 b$ then ensures that all constraints are simultaneously satisfied by the interpolated flows calculated in step 3c.

The consideration only of truly interpolated flows and constraints, i.e., the limitation of $\lambda^{*}$ to $[0,1]$, is justified by the observation that both working points constitute extreme cases of the model: Point A represents maximum flows because no node supply constraints are applied. Assuming that increasing the flows in an intersection causes more mutual obstructions and hence a tightening of the node supply constraints, point A also represents the tightest possible constraints. Vice versa, point $B$, resulting from these tightest constraints, represents particularly small flows B, which in turn result in utmost weak constraints B. 


$\overline{\text { Algorithm } 3 \text { Approximate solution procedure }}$

1. calculate working point A:

(a) $\mathbf{q}^{\mathrm{A}}=\operatorname{INM}(\boldsymbol{\Delta}, \boldsymbol{\Sigma})$

(b) $\widehat{\Delta}^{\mathrm{A}}=\min \left\{\boldsymbol{\Delta}, \widehat{\Delta}\left(\mathbf{q}^{\mathrm{A}}\right)\right\}$

2. calculate working point $\mathrm{B}$ :
(a) $\mathbf{q}^{\mathrm{B}}=\operatorname{INM}\left(\widehat{\boldsymbol{\Delta}}^{\mathrm{A}}, \boldsymbol{\Sigma}\right)$
(b) $\widehat{\Delta}^{\mathrm{B}}=\min \left\{\boldsymbol{\Delta}, \widehat{\Delta}\left(\mathbf{q}^{\mathrm{B}}\right)\right\}$

3. solve linearized model with $\mathbf{q}(\lambda)=\mathbf{q}^{\mathrm{B}}+\lambda\left(\mathbf{q}^{\mathrm{A}}-\mathbf{q}^{\mathrm{B}}\right)$ and $\widehat{\Delta}(\lambda)=\widehat{\Delta}^{\mathrm{B}}+$ $\lambda\left(\widehat{\Delta}^{\mathrm{A}}-\widehat{\Delta}^{\mathrm{B}}\right)$ :

(a) for all $i=1 \ldots$, evaluate

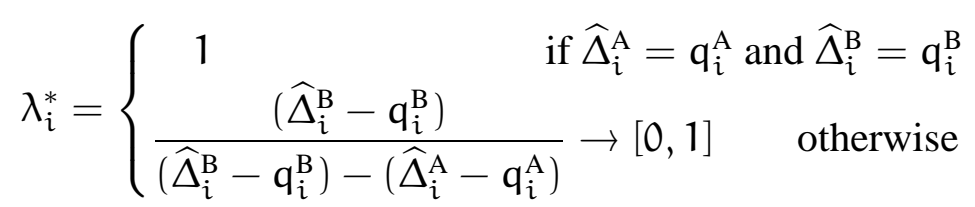

(b) $\lambda^{*}=\min _{i=1 \ldots I} \lambda_{i}^{*}$

(c) $\mathbf{q}=\mathbf{q}^{\mathrm{B}}+\lambda^{*}\left(\mathbf{q}^{\mathrm{A}}-\mathbf{q}^{\mathrm{B}}\right)$

\section{Experiments}

This section tests the INMC in two settings. First, Subsection 4.1 compares the model in a synthetic setting to a detailed traffic microsimulator. The objective of this study is to investigate the model in a complex configuration for which no real data is available. Second, Subsection 4.2 applies the model to a real intersection and compares it to real data.

\subsection{Synthetic experiment}

The same network as used in Section 2.4 is considered, cf. Figure 3, and all previous modeling assumptions are maintained. Beyond this, the INM is now supplemented with two node supply constraints: One constraint affects the left-turning 
fraction of the northbound major stream because of the oncoming southbound major stream, and one constraint affects the minor stream, which crosses both major streams. The resulting INMC is compared to the detailed traffic microsimulator AIMSUN NG 5.1.10 (TSS Transport Simulation Systems, 2006).

Link flows are captured here and in the following using a cell-transmission model (CTM) (Daganzo, 1994) with a trapezoidal fundamental diagram. The trapezoidal CTM has found various applications, e.g., in freeway ramp metering, signal optimization, and traffic state estimation (Friedrich and Almasri, 2006; Feldman and Maher, 2002; Sun et al., 2003; Tampere and Immers, 2007), and has been subject to thorough experimental validations (e.g., Brockfeld and Wagner, 2006; Munoz et al., 2006, 2004). Early network implementations are NETCELL (Cayford et al., 1997), and the model of Lee (1996); Ziliaskopoulos and Lee (1997). The latter allows for urban intersections but relies on a sole recombination of simple merge and diverge building blocks and applies only to signalized intersections.

The following parameters are obtained by manual calibration against the microsimulator: All links but the minor street's entry link have a free flow speed of $50 \mathrm{~km} / \mathrm{h}$, a flow capacity of $2340 \mathrm{veh} / \mathrm{h}$, a backward wave speed of $16 \mathrm{~km} / \mathrm{h}$, and a maximum density of $200 \mathrm{veh} / \mathrm{km}$. The minor street's entry is stop-controlled. AIMSUN captures the effect of the stop sign by physically simulating a full stop of every vehicle. The macroscopic model reflects this through a flow capacity of $518 \mathrm{veh} / \mathrm{h}$ and a maximum velocity of $32.4 \mathrm{~km} / \mathrm{h}$ (whereas the free flow speed in AIMSUN is $50 \mathrm{~km} / \mathrm{h}$ ). The backward wave speed and the maximum density are the same as for all other links.

The links of the major street are $150 \mathrm{~m}$ long; the links of the minor street have a length of $100 \mathrm{~m}$. All streets are modeled in AIMSUN as 1-lane urban roads (volume delay function 38786: VDF 25). The AIMSUN traffic demand consists of the vehicle type "car" only. For easy reproducibility, all microscopic vehicle parameters (e.g., maximum acceleration and deceleration, minimum distance between vehicles, etc.) are set to their AIMSUN default values. (The ability of the INMC to reproduce real situations is demonstrated in the next subsection.)

The external demand inflow patterns are shown in Figure 7, where the bold lines represent macroscopic values in the INMC and the thin lines are the according AIMSUN flows, which are averaged over 10 simulations but still exhibits substantial vehicle discretization noise. In AIMSUN, the inflow patterns are generated by adding traffic light controlled links upstream of each ingoing link of the original network (cycle time $90 \mathrm{~s}$, major street: green time $24 \mathrm{~s}$, minor street: green time $9 \mathrm{~s})$. In the INMC, piecewise linear demand flow patterns are found to reflect the AIMSUN inflows well. The figure clearly reflects the $90 \mathrm{~s}$ cycle time of the upstream traffic lights, which all turn green towards the central intersection at the 
same time. Furthermore, the constellation of link lengths, maximum velocities, and stop sign-induced delay is such that all platoons reach the intersection after approximately $11 \mathrm{~s}$.

The left-turning portion of the northbound stream yields to the straight portion of the southbound stream. The demand constraint function capturing this effect is obtained from the German Highway Capacity Manual (HBS), Chapter "Unsignalized Intersections" (Forschungsgesellschaft für Strassen und Verkehrswesen, 2001). Its functional form is

$$
\widehat{\Delta}_{S}\left(q_{N}^{\text {in }}\right)=\frac{1}{t_{f, S}} \exp \left[-q_{N}^{\text {in }}\left(t_{g, S}-\frac{t_{f, S}}{2}\right)\right]
$$

where $q_{N}^{\text {in }}$ is the node inflow rate of the southbound stream, $t_{g, S}$ is the minimum time gap between two southbound vehicles that allows one left-turning vehicle to enter the intersection, and $t_{f, S}$ is the minimum follow-up time between two left-turning vehicles, with a recommended value of $\frac{13}{21} t_{\mathrm{g}, \mathrm{S}}$. These parameters are identified by manual calibration against AIMSUN: $t_{\mathrm{g}, \mathrm{S}}=8.4 \mathrm{~s}$ and $t_{f, S}=5.2 \mathrm{~s}$. Note that here and in the following, the HBS is used only exemplary in order to obtain concrete node supply constraints. The INMC framework is not limited to specifications from the HBS.

The minor road is stop-controlled and yields to the major streams. The effect of having to stop at all is captured by choosing a relatively small flow capacity and a small maximum velocity as described before. Beyond this, the minor stream yields to the major streams. The according demand constraint function is again obtained from Forschungsgesellschaft für Strassen und Verkehrswesen (2001):

$$
\widehat{\Delta}_{E}\left(q_{S}^{\text {in }}, q_{N}^{\text {in }}\right)=\frac{p_{0, S}}{t_{f, E}} \exp \left[-\left(q_{S}^{\text {in }}+q_{N}^{\text {in }}\right)\left(t_{g, E}-\frac{t_{f, E}}{2}\right)\right]
$$

where $t_{g, E}$ and $t_{f, E}$ are again the minimum gap and the follow up time, $q_{S}^{\text {in }}$ and $q_{\mathrm{N}}^{\text {in }}$ are the conflicting major flows, and $p_{0, S}$ is the probability that the left-turning traffic on the major street operates in a queue-free state. Again, the parameters are identified by manual calibration: $t_{\mathrm{g}, \mathrm{E}}=9 \mathrm{~s}, \mathrm{p}_{\mathrm{O}, \mathrm{S}}=0.15$, and $\mathrm{t}_{\mathrm{f}, \mathrm{E}}=8 \mathrm{~s}$ according to the recommendation $t_{f, E}=\frac{8}{9} t_{g, E}$.

The chosen setting is such that the capacity of the westbound merge has no limiting effect on the intersection's throughput, which allows to explain all flow interactions by the node supply constraints. This also enables a comparison of the exact and the approximate solution procedure for the INMC, cf. Section 3.3: The constraints follow a hierarchical ordering (flow from the north precedes all other flows, flow from the south precedes flow from the east) such that the exact solution procedure can be applied. The approximate solution method is always applicable. 



Figure 7: Comparison of microscopic and macroscopic demand curves The figure contains three diagrams, one for each ingoing link of the intersection. The thin noisy curve is the microscopic AIMSUN demand, and the respective fat smooth curve is the macroscopic demand. The AIMSUN curve is averaged over 10 runs of the microsimulator but still exhibits substantial vehicle discretization noise. 
Figure 8 shows the simulation results for both AIMSUN and the INMC. The figure contains three diagrams, one for each ingoing link's flow discharge into the intersection. The thin noisy curve is the AIMSUN output, which is averaged over 10 simulations, and the respective thick smooth curve is obtained from the INMC (where the exact and the approximate solution procedure yield visually identical results).

Cars coming from the north traverse the intersection without interruption. One observes a shift of the respective demand profile by approximately $11 \mathrm{~s}$, which is the time it takes the demand to travel from the network entry to the central node. The flow coming from the south is equally delayed, but once it reaches the intersection, its left-turning portion is quickly suppressed by the higher-ranking southbound flow, which effectively holds up the entire northbound flow (remember that all roads have a single lane only). Only when the southbound flow ceases, the northbound flow can continue, which it does at maximum capacity. Finally, the minor stream going from east to west is held up until both major streams have passed the intersection. Some minor flow makes it through when the southbound flow ceases and the northbound flow has not yet reached its maximum value. All queues recover within a 90 s cycle.

A comparison between AIMSUN and the INMC shows that the microscopic traffic phenomena (no interruption of the southbound flow; temporary queuing of both other flows) are well captured by the macroscopic model. The uninterrupted outflow from the north is somewhat wider in AIMSUN than in the macroscopic model because the latter does not capture platoon dispersion, which, however, is not so much of an intersection modeling problem but rather an inherent features of all first order models. ${ }^{6}$ The node supply constraints generate flow reductions that are consistent with AIMSUN's representation of the respective vehicle interactions. The recovery of the southern queue is faster in the macroscopic model than in the microsimulator because the KWM postulates infinite vehicle accelerations. This, again, is an inherent feature of all first order models.

The visual impression of the flow curves presented above is supported by a quantitative comparison of the average densities and delays on all links in the network. The results are shown in Table 3 and exhibit a remarkable accuracy. In particular, the delay estimates of the macroscopic model (computed from cumulative flow curves) almost perfectly reproduce the outputs of the far more complicated microsimulator. However, it should be noted that the default acceleration values in

\footnotetext{
${ }^{6}$ Dispersion at the downstream end of a platoon could be captured by choosing a truly concave flow demand function $\Delta$. Dispersion at a platoon's upstream end, however, cannot be captured by a first order model: Flows at lower densities are never slower than flows at higher densities such that any platoon tail will eventually catch up with the platoon main body and transform into a density discontinuity at its upstream end.
} 



Figure 8: Comparison of AIMSUN and ECTM simulation results

The figure contains three diagrams, one for each ingoing link of the intersection. The thin noisy curve is the AIMSUN output, and the respective fat smooth curve is obtained from the ECTM. The AIMSUN curve is averaged over 10 runs of the microsimulator but still exhibits substantial vehicle discretization noise. 
Table 3: Quantitative comparison of AIMSUN and INMC

\begin{tabular}{|r||c|c|c||c|c|c|}
\hline \multicolumn{1}{|c||}{\multirow{2}{*}{ link }} & \multicolumn{3}{c||}{ density [veh/km] } & \multicolumn{3}{c|}{ delay [s/veh] } \\
\cline { 2 - 7 } & $\begin{array}{c}\text { AIM- } \\
\text { SUN }\end{array}$ & $\begin{array}{c}\text { INMC } \\
\text { (exact) }\end{array}$ & $\begin{array}{c}\text { INMC } \\
\text { (approx.) }\end{array}$ & $\begin{array}{c}\text { AIM- } \\
\text { SUN }\end{array}$ & $\begin{array}{c}\text { INMC } \\
\text { (exact) }\end{array}$ & $\begin{array}{c}\text { INMC } \\
\text { (approx.) }\end{array}$ \\
\hline \hline S, ingoing & 28.6 & 31.7 & 31.6 & 23 & 23 & 23 \\
\hline E, ingoing & 24.5 & 29.3 & 29.2 & 59 & 57 & 56 \\
\hline N, ingoing & 11.7 & 10.1 & 10.1 & 3 & 0 & 0 \\
\hline N, outgoing & 4.5 & 4.9 & 4.9 & 1 & 0 & 0 \\
\hline W, outgoing & 14.5 & 13.0 & 13.0 & 1 & 0 & 0 \\
\hline S, outgoing & 4.7 & 5.0 & 5.0 & 1 & 0 & 0 \\
\hline
\end{tabular}

AIMSUN are quite high, which biases the comparison in favor of the KWM. The next subsection provides more insight into this issue. Finally, it also is noteworthy that the exact and the approximate solution procedure yield almost identical results.

\subsection{Real experiment}

This experiment considers a real intersection in Braunschweig, Germany, which is shown in Figure 9 (left). The intersection consists of a two-lane main road (Altewiekring) in north/south direction and a minor one-lane road (Kastanienallee) in east/west direction. All approaches have separate left-turn pockets. The intersection is fixed-time controlled with a two-phase signal sequence and a cycle length of $85 \mathrm{~s}$. The first phase corresponds to a green time of $44 \mathrm{~s}$ for the main road; the second phase corresponds to a green time of $24 \mathrm{~s}$ for the minor road. The intergreen time is $9 \mathrm{~s}$ between the first and second phase and $8 \mathrm{~s}$ between the second and first phase, respectively.

The analysis focuses on the interaction of the north/south main traffic streams; since they are decoupled from the minor streams through the signaling, an incorporation of the minor streams would not add complexity to the node modeling. Also, the turning pockets decouple the left-turning stream from the straight and right-turning stream of the same approach. The interactions of a single leftturning stream with all of its conflicting streams can therefore be investigated in isolation. In consequence, the considered setting is constrained to the left-turning main stream coming from the north and the straight and right-turning main streams coming from the south. Figure 9 (right) shows how this subsystem is modeled.

The node model has three ingoing lanes and two outgoing lanes, which are mod- 



Figure 9: Braunschweig intersection Altewiekring/Kastanienallee Left: real intersection (from Google Maps). Right: modeled subset.

eled with the CTM. Five cells constitute boundaries to the node: $P_{N}$ represents the left-turning stream coming from the north. $\mathrm{P}_{\mathrm{S} 1}$ represents the middle lane of the northbound stream coming from the south. $P_{\mathrm{S} 2}$ represents the right lane coming from the south, diverging north and east. $P_{\mathrm{S} 1}$ and the northbound portion of $\mathrm{P}_{\mathrm{S} 2}$ merge into $S_{N}$. The left-turning vehicles from $P_{N}$ merge with the right-turning flow from $P_{\mathrm{S} 2}$ into $S_{\mathrm{E}}$. The left-turning stream yields to the exit flow of $\mathrm{P}_{\mathrm{S} 1}$ and $\mathrm{P}_{\mathrm{S} 2}$.

The model is manually calibrated against data from video recordings of 25 cycles during the evening peak hour of March 10, 2010. The link upstream arrivals and the node inflows are evaluated in $5 \mathrm{~s}$ intervals, which divides the cycle duration of $85 \mathrm{~s}$ into 17 intervals.

The real link upstream arrivals are macroscopically approximated by constant inflow rates per cycle, starting (ending) $40 \mathrm{~s}$ before the green phase (red phase) for the $P_{\mathrm{S} 1}$ and $P_{\mathrm{S} 2}$ lanes and starting (ending) $34 \mathrm{~s}$ before the green phase (red phase) for the $P_{N}$ lane. This yields realistic results for all but three cycles of $P_{S 1}$ and $P_{S 2}$, where the respective inflows from upstream cease during the green period. This is modeled through a piecewise constant inflow profile that drops to zero consistently with the video footage. It should be noted that in a network model these 
manually extracted boundary conditions would be defined through upstream and downstream node models.

The model is solved with Algorithm 2. The simulation time step length is one second. All cells have a free flow speed of $45 \mathrm{~km} / \mathrm{h}$ (the local speed limit is $50 \mathrm{~km} / \mathrm{h}$, but hardly a vehicle reaches this speed), a resulting cell length of $12.5 \mathrm{~m}$, and a jam density of $175 \mathrm{veh} / \mathrm{km}$ (estimated from video). Assuming a triangular fundamental diagram, the backward wave speed is $17.1 \mathrm{~km} / \mathrm{h}$. The flow capacity of all cells but $S_{\mathrm{E}}$ is $2160 \mathrm{veh} / \mathrm{h}(0.6 \mathrm{veh} / \mathrm{s}$; this corresponds to the largest observed average flow per cycle). The flow capacity of $S_{E}$ is $1200 \mathrm{veh} / \mathrm{h}(0.33 \mathrm{veh} / \mathrm{s})$ because pedestrians frequently cross $S_{E}$ in north/south direction. The video footage reveals a turning fraction of 0.53 from $P_{\mathrm{S} 2}$ towards $S_{\mathrm{N}}$ and 0.47 towards $S_{\mathrm{E}}$. All inflow priorities are set to one. This is plausible for $P_{\mathrm{S} 1}$ and $P_{\mathrm{S} 2}$ because they merge coequally into $S_{N}$. The priority value of $P_{N}$ cannot be estimated from the data because whenever there could have been a competition with $P_{S 2}$ for $S_{E}$, the flow from $P_{N}$ is already held back inside of the intersection by $P_{S 1}$ and the straight portion of $\mathrm{P}_{\mathrm{S} 2}{ }^{7}$

The model is supplemented with one node supply constraint that captures the yielding of the left-turning flow from $\mathrm{P}_{\mathrm{N}}$. The according demand constraint function is modeled symmetrically to (30):

$$
\widehat{\Delta}_{N}\left(q_{S}^{\text {in }}\right)=\frac{1}{t_{f, N}} \exp \left[-q_{S}^{\text {in }}\left(t_{g, N}-\frac{t_{f, N}}{2}\right)\right]
$$

with $q_{S}^{\text {in }}=q_{S 1}^{\text {in }}+q_{S 2}^{\text {in }}$ and, like before, $t_{f, N}=\frac{13}{21} t_{g, N}$. The video footage reveals that the discharge from $P_{N}$ given a zero conflicting flow $q_{S}^{\text {in }}$ is much higher during phase one than during the inter-green phase after phase one in otherwise identical conditions. A plausible explanation is that in the inter-green time, when the major stream already has red signaling, there are no vehicles approaching from the south that could close an existing gap such that the left-turning vehicles discharge more calmly than during phase one. This effect is captured with a time- and flowdependent $t_{g, N}$ parameter, which is $7.2 \mathrm{~s}$ in most cases, rapidly goes to $4.85 \mathrm{~s}$ if $\mathrm{q}_{\mathrm{S}}^{\text {in }}$ becomes zero during phase one, and to $11.63 \mathrm{~s}$ during the $18 \mathrm{~s}$ after phase one. All values are higher than the standard recommendation of $4.2 \mathrm{~s}$ for German intersections (Forschungsgesellschaft für Strassen und Verkehrswesen, 2001) because they also compensate for the unlimited vehicle acceleration in the KWM.

\footnotetext{
${ }^{7}$ This reflects a general identifiability issue: If certain conflicts (or signal settings) render the occurrence of other conflicts a rare event, the parameters describing the latter cannot be estimated from data. However, such parameters also rarely take effect in the model, such that the transferal of guidelines from the literature that were developed for similar situations may yield satisfactory solutions.
} 
Figure 10 shows the real and modeled intersection inflows from $\mathrm{P}_{\mathrm{S} 1}, \mathrm{P}_{\mathrm{S} 2}$, and $\mathrm{P}_{\mathrm{N}}$, all averaged in $5 \mathrm{~s}$ time bins. The thin lines represent the real data, and the thick lines represent the model. One clearly recognizes the periodic rhythm of the intersection. The southern node inflows $q_{S 1}^{\text {in }}$ and $q_{S 2}^{\text {in }}$ have a two-step shape in most cycles: First, the respective queue discharges at the capacity limit. Once it is dissipated, the flow rate drops to the exogenous upstream arrival rate. The node inflow from $P_{N}$ is delayed until the flows from $P_{S 1}, P_{S 2}$ have ceased. The light gray boxes between seconds 1461 and 1526 indicate a situation where spillback occurs from the downstream end of $S_{\mathrm{E}}$. Modeling this effect by reducing the outflow capacity of $S_{\mathrm{E}}$ accordingly affects both $\mathrm{P}_{\mathrm{S} 2}$ and $\mathrm{P}_{\mathrm{N}}$ : The flow from $\mathrm{P}_{\mathrm{N}}$ is held back while the right-turning portion of $P_{\mathrm{S} 2}$ uses up up the remaining space in $S_{E}$, such that the node inflow from $P_{N}$ drops to almost zero. Overall, the macroscopic flows capture the trend of the real data very well.

Figure 11 compares the real and modeled queue on the $P_{N}$ lane. For this, the number of all vehicles with a velocity of $6 \mathrm{~km} / \mathrm{h}$ or less is compared to the number of queued vehicles in the video footage two times per cycle, once at the beginning of the green time of phase one and once when the last left-turning vehicle has cleared the intersection. Again, the thin line represents the real data and the thick line represents the model. Both the build-up and the dissipation of the peak hour queue are well captured. This is particularly meaningful because the $P_{N}$ approach is over-saturated during much of the peak hour such that any deviation between the curves represents a cumulation of flow modeling errors over several cycles. ${ }^{8}$ From the fact that the true and the modeled queue do not drift apart, one can conclude that there is no systematic bias the modeled flows. The average real queue size is 3.72 vehicles, compared to an average modeled queue size of 3.33 vehicles, which constitutes a minor under-estimation by less than half a vehicle.

Finally, Figure 12 shows the real and modeled travel times as experienced by the left-turning vehicles when leaving $S_{\mathrm{E}}$. The real travel time is computed from number plate recognition sensors, with the first sensor being located at the upstream end of $P_{N}$ and the second sensor at the downstream end of $S_{E}$. It is again represented by the thin solid line. A naive computation of the modeled travel time by comparing the respective cumulative flow curves results in a systematic underestimation of the travel time, for which two reasons are identified: (i) the point-like node model neglects the time it takes to cross the intersection; (ii) the

\footnotetext{
${ }^{8}$ At the beginning of phase one's green time, two or three left-turning vehicles enter the intersection, stop near its center, and wait for sufficiently long gaps in the opposite right-of-way flow. If there are no or only very few such gaps, these vehicles have to wait until the green time of the major stream ends and can only then pass the intersection. The traffic demand of the left-turning flow during the peak hour is sometimes much higher than two or three vehicles per cycle and some vehicles wait up to 5 cycles only to enter the intersection.
} 


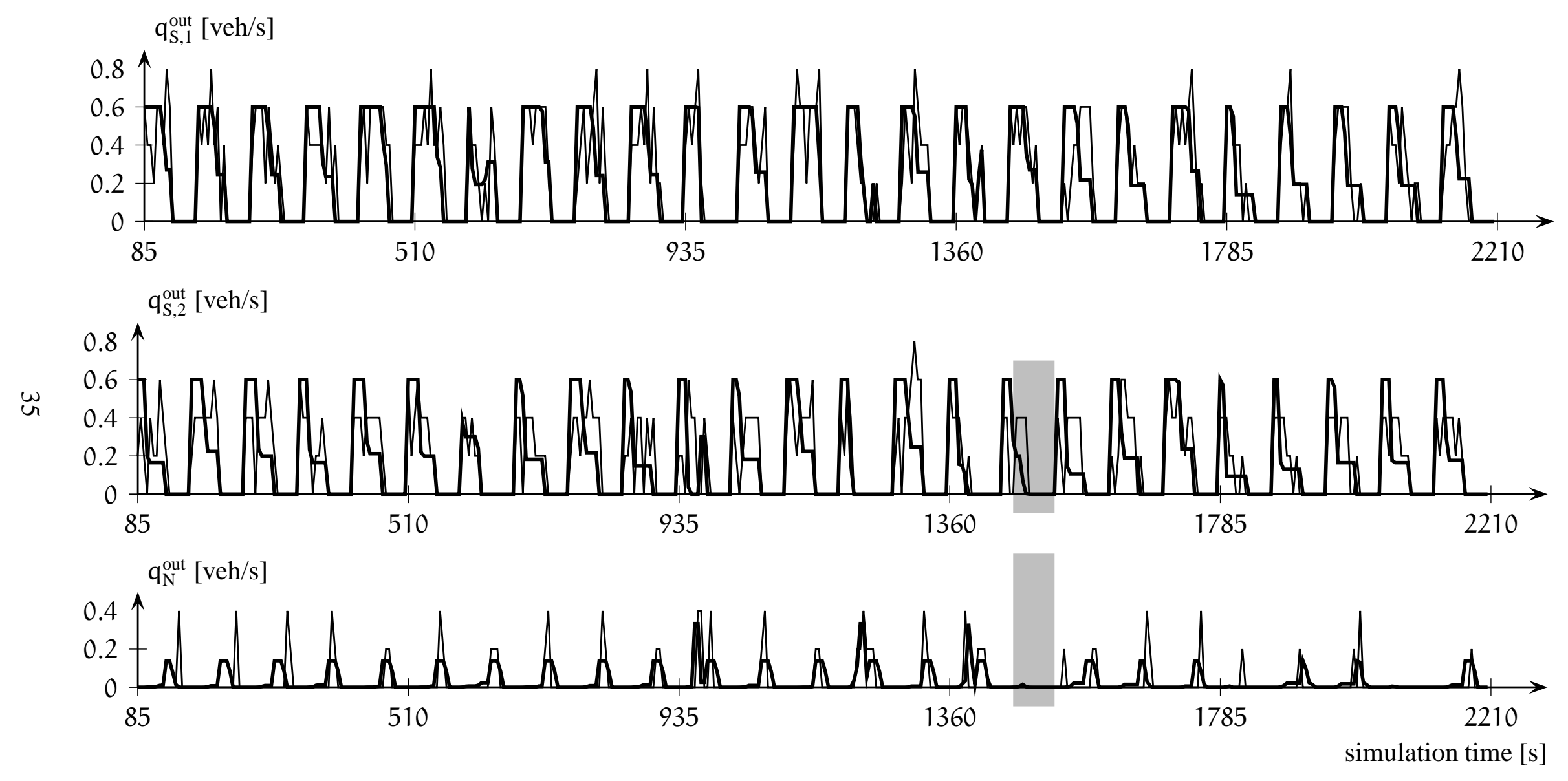

Figure 10: Real and modeled intersection inflows 




Figure 11: Real and modeled queue sizes

KWM neglects the finite acceleration of the left-turning vehicles when leaving the intersection. This error is therefore corrected by computing only the travel time through $\mathrm{P}_{\mathrm{N}}$ from the respective cumulative flows and then adding the average time it takes an otherwise unhindered vehicle to proceed until the end of the $\mathrm{S}_{\mathrm{E}}$, which is $26 \mathrm{~s}$. The result is represented by the thick curve. Clearly, it captures the overall rhythm of the evening peak hour well, but it also leaves some room for improvement. A more powerful correction than a constant offset would be to resort to a model that explicitly captures finite accelerations (e.g., Lebacque, 1984, 2003). However, it should also be noted that this example is particularly sensitive to modeling imprecisions: Since the left-turning vehicles wait up to 5 cycles until they enter the intersection, their travel time effectively incorporates the modeling errors during many cycles. The average real travel time is $168 \mathrm{~s}$, compared to an average modeled travel time of $161 \mathrm{~s}$; this mild under-estimation is consistent with the minor under-estimation of the queue size in the $\mathrm{P}_{\mathrm{N}}$ lane.

In summary, these experiments clearly demonstrate that the INMC is able to capture the flows in a complex real intersection very well.

\section{Summary}

This article contributes to the modeling and simulation of complex urban traffic intersections from a methodological, computational, and empirical point of view. 




Figure 12: Real and modeled travel times

Methodologically, there are two major contributions. First, the incremental transfer (IT) principle of Daganzo et al. (1997) is carried over to the modeling of urban intersections. The resulting incremental node model (INM) covers a rich subset of the generic node model (GNM) class of Tampere et al. (forthcoming) and comprises many models proposed so far in the literature. Second, the INM is supplemented with a fixed point condition that allows to incorporate rich node supply constraints that may result from signaling and/or conflicts (INMC). An insightful uniqueness analysis reveals the limitations of point-like and flow maximizing node models.

Computationally, specifications of the INM are described that allow for very simple and efficient simulation procedures. Furthermore, two algorithms for the solution of the more complex INMC are presented. The first method is exact but assumes that all intersection inflows can be ordered in some priority-related manner. The second method applies to arbitrary intersections but may yield only approximate solutions. However, no significant difference between the exact and the approximate solution procedure is observed in the experimental results.

Empirically, experiments with both synthetic and real data are conducted. The results demonstrate the correctness and practical relevance of the INMC. They show that it is possible to capture complex flow interactions in urban intersections with macroscopic node models. The comparison with real data also reveals that the infinite accelerations of the KWM constitute its major shortcoming when modeling urban intersections. 
Important topics for future research comprise the following items:

- Exploitation of the general INM specification through non-constant flow transfer rates.

- Relaxation of the requirement of global flow maximization in the GNM, possibly through an alternative fixed point formulation.

- Relaxation of the FIFO assumption. This effort would likely benefit from the original IT principle for multi-lane traffic flow.

- Compensating for the infinite KWM accelerations inside the node.

- Development of systematic calibration approaches and data collection strategies for the estimation of concrete INM(C) instances.

- Collection of more empirical evidence and testing of new INMC instances.

\section{Acknowledgments}

Peter Wagner gave very helpful comments on an early version of this article. Bernhard Friedrich provided valuable support and technical equipment for the experiments.

\section{References}

Adamo, V., Astarita, V., Florian, M., Mahut, M. and Wu, J. (1999). Modelling the spill-back of congestion in link based dynamic network loading models: a simulation model with applications, in A. Ceder (ed.), Proceedings of the 14th International Symposium on Transportation and Traffic Theory, Pergamon, Jerusalem, Israel, pp. 555-573.

Ansorge, R. (1990). What does the entropy condition mean in traffic flow theory, Transportation Research Part B 24(2): 133-143.

Astarita, V., Er-Rafia, K., Florian, M., Mahut, M. and Velan, S. (2001). A comparison of three methods for dynamic network loading, Transportation Research Record 1771: 179-190.

Ben-Akiva, M., Bierlaire, M., Burton, D., Koutsopoulos, H. and Mishalani, R. (2001). Network state estimation and prediction for real-time transportation management applications, Networks and Spatial Economics 1: 293-318. 
Bliemer, M. (2007). Dynamic queuing and spillback in an analytical multiclass dynamic network loading model, Transportation Research Record 2029: 14 21.

Brackstone, M. and McDonald, M. (1999). Car-following: a historical review, Transportation Research Part F 2(4): 181-196.

Brockfeld, E. and Wagner, P. (2006). Validating microscopic traffic flow models, Proceedings of the 9th IEEE Intelligent Transportation Systems Conference, Toronto, Canada, pp. 1604-1608.

Buisson, C., Lebacque, J. and Lesort, J. (1996). STRADA. A discretized macroscopic model of vehicular traffic flow in complex networks based on the Godunov scheme., Symposium on Modelling, Analysis and Simulation, held at CESA 1996 IMACS Multiconference, Vol. 2, Lille, France, pp. 976-981.

Buisson, C., Lebacque, J., Lesort, J. and Mongeot, H. (1996). The STRADA model for dynamic assignment, Proceedings of the 1996 ITS Conference, Orlando, USA.

Buisson, C., Lesort, L. and Lebacque, J. (1995). Macroscopic modelling of traffic flow and assignment in mixed networks, in P. Pahl and H. Werner (eds), Computing in Civil and Building Engineering, Balkema, Rotterdam, The Netherlands, pp. 1367-1374.

Cayford, R., Lin, W.-H. and Daganzo, C. (1997). The NETCELL simulation package: technical description, California PATH research report UCB-ITS-PRR-9723, University of California, Berkeley.

Cheu, R., Martinez, J. and Duran, C. (2009). A cell transmission model with lane changing and vehicle tracking for port of entry simulations, Transportation Research Record 2124: 241-248.

Chrobok, R., Pottmeier, A., Wahle, J. and Schreckenberg, M. (2003). Traffic forecast using a combination of on-line simulation and traffic data, in M. Fukui, Y. Sugiyama, M. Schreckenberg and D. Wolf (eds), Traffic and Granular Flow '01, Springer, pp. 345-350.

Coclite, G., Garavello, M. and Piccoli, B. (2005). Traffic flow on a road network, SIAM Journal on Mathematical Analysis 36(6): 1862-1886.

Daganzo, C. (1994). The cell transmission model: a dynamic representation of highway traffic consistent with the hydrodynamic theory, Transportation Research Part B 28(4): 269-287. 
Daganzo, C. (1995a). The cell transmission model, part II: network traffic, Transportation Research Part B 29(2): 79-93.

Daganzo, C. (1995b). A finite difference approximation of the kinematic wave model of traffic flow, Transportation Research Part B 29(4): 261-276.

Daganzo, C. (2006). In traffic flow, cellular automata = kinematic waves, Transportation Research Part B 40(5): 396-403.

Daganzo, C., Lin, W.-H. and del Castillo, J. (1997). A simple physical principle for the simulation of freeways with special lanes and priority vehicles, Transportation Research Part B 31(2): 103-125.

De Palma, A. and Marchal, F. (2002). Real cases applications of the fully dynamic METROPOLIS tool-box: an advocacy for large-scale mesoscopic transportation systems, Networks and Spatial Economics 2: 347-369.

Elsevier (accessed 2010). Scopus web site, http://www.scopus.com.

Feldman, O. and Maher, M. (2002). The optimisation of traffic signals using a cell transmission model, Proceedings of the 9th Meeting of the EURO Working Group on Transportation, Bari, Italy, pp. 503-507.

Flötteröd, G. (2008). Traffic State Estimation with Multi-Agent Simulations, $\mathrm{PhD}$ thesis, Berlin Institute of Technology, Berlin, Germany.

Flötteröd, G. and Nagel, K. (2005). Some practical extensions to the cell transmission model, Proceedings of the 8th IEEE Intelligent Transportation Systems Conference, Vienna, Austria, pp. 510-515.

Forschungsgesellschaft für Strassen und Verkehrswesen (2001). Handbuch für die Bemessung von Strassenverkehrsanlagen, FSGV Verlag GmbH, Köln.

Friedrich, B. and Almasri, E. (2006). A new method for offset optimization in urban road networks, Proceedings of the 11th Meeting of the Euro Working Group Transportation, Bari, Italy.

Gentile, G., Meschini, L. and Papola, N. (2007). Spillback congestion in dynamic traffic assignment: a macroscopic flow model with time-varying bottlenecks, Transportation Research Part B 41(10): 1114-1138.

Greenshields, B. (1935). A study of traffic capacity, Proceedings of the Annual Meeting of the Highway Research Board, Vol. 14, pp. 448-477. 
Herty, M. and Klar, A. (2003). Modeling, simulation, and optimization of traffic flow networks, SIAM Journal on Scientific Computing 25(3): 1066-1087.

Holden, H. and Risebro, N. (1995). A mathematical model of traffic flow on a network of unidirectional roads, SIAM Journal on Mathematical Analysis 26(4): 999-1017.

Hoogendoorn, S. and Bovy, P. (2001). State-of-the-art of vehicular traffic flow modelling, Proceedings of the Institution of Mechanical Engineers. Part I: Journal of Systems and Control Engineering 215(4): 283-303.

Jin, W. and Zhang, H. (2003). On the distribution schemes for determining flows through a merge, Transportation Research Part B 37(6): 521-540.

Jin, W. and Zhang, H. (2004). A multicommodity kinematic wave simulation model of network traffic flow, Transportation Research Record 1883: 59-67.

Kurzhanskiy, A. and Varaiya, P. (2010). Using Aurora road network modeler for active traffic management, Proceedings of the 2010 American Control Conference, Baltimore, MD, USA.

Kuwahara, M. and Akamatsu, T. (2001). Dynamic user optimal assignment with physical queues for a many-to-many OD pattern, Transportation Research Part $B$ 35(5): 461-479.

Laval, J. and Daganzo, C. (2006). Lane-changing in traffic streams, Transportation Research Part B 40(3): 251-264.

Lawson, T., Lin, W. and Cassidy, M. (1999). Validation of the incremental transfer model, California PATH working paper UCB-ITS-PWP-99-16, Institute of Transportation Studies, University of California, Berkeley.

Lebacque, J. (1984). Semi-macroscopic simulation of urban traffic, Proceedings of the International AMSE Conference "Modelling \& Simulation", Vol. 4, Minneapolis, USA, pp. 273-292.

Lebacque, J. (1996). The Godunov scheme and what it means for first order traffic flow models, in J.-B. Lesort (ed.), Proceedings of the 13th International Symposium on Transportation and Traffic Theory, Pergamon, Lyon, France.

Lebacque, J. (2003). Intersection modeling, application to macroscopic network traffic flow models and traffic management, Proceedings of TFG'03 Traffic and Granular Flow Conference, Delft, The Netherlands. 
Lebacque, J. and Khoshyaran, M. (2005). First-order macroscopic traffic flow models: intersection modeling, network modeling, in H. Mahmassani (ed.), Proceedings of the 16th International Symposium on Transportation and Traffic Theory, Elsevier, Maryland, USA, pp. 365-386.

Lebacque, J. and Koshyaran, M. (2002). First order macroscopic traffic flow models for networks in the context of dynamic assignment, in M. Patrikson and M. Labbé (eds), Transportation Planning, Kluwer, pp. 119-139.

Lebacque, J. and Lesort, J. (1999). Macroscopic traffic flow models: a question of order, in A. Ceder (ed.), Proceedings of the 14th International Symposium on Transportation and Traffic Theory, Pergamon, Jerusalem, Israel, pp. 3-25.

Lee, S. (1996). A cell transmission based assignment-simulation model for integrated freeway/surface street systems, Master thesis, Ohio State University.

Lighthill, M. and Witham, J. (1955). On kinematic waves II. a theory of traffic flow on long crowded roads, Proceedings of the Royal Society A 229: 317-345.

Mahmassani, H. S. (2001). Dynamic network traffic assignment and simulation methodology for advanced system management applications, Networks and Spatial Economics 1(3/4): 267-292.

Munoz, L., Sun, X., Horowitz, R. and Alvarez, L. (2006). A piecewise-linearized cell transmission model and parameter calibration methodology, Proceedings of the 85. Annual Meeting of the Transportation Research Board, Washington, DC, USA.

Munoz, L., Sun, X., Sun, D. and nd R. Horowitz, G. G. (2004). Methodological calibration of the cell transmission model, Proceedings of the American Control Conference, Denver, Colorado, pp. 798-803.

Nagel, K. and Nelson, P. (2005). A critical comparison of the kinematic-wave model with observational data, in $\mathrm{H}$. Mahmassani (ed.), Proceedings of the 16th International Symposium on Transportation and Traffic Theory, Elsevier, Maryland, USA, pp. 145-163.

Nelson, P. and Kumar, N. (2006). Point constriction, interface, and boundary conditions for kinematic-wave model, Transportation Research Record 1965: 6069.

$\mathrm{Ni}$, D. and Leonard, J. (2005). A simplified kinematic wave model at a merge bottleneck, Applied Mathematical Modelling 29(11): 1054-1072. 
Nie, Y. (2010). personal communication.

Nie, Y., Ma, J. and Zhang, H. (2008). A polymorphic dynamic network loading model, Computer-Aided Civil and Infrastructure Engineering 23(2): 86-103.

Nökel, K. and Schmidt, M. (2002). Parallel DYNEMO: meso-scopic traffic flow simulation on large networks, Networks and Spatial Economics 2(4): 387-403.

Pandawi, S. and Dia, H. (2005). Comparative evaluation of microscopic carfollowing behavior, IEEE Transactions on Intelligent Transportation System 6(3): 314-325.

Payne, H. (1971). Models of freeway traffic and control, Mathematical Models of Public Systems, Vol. 1, Simulation Council, La Jolla, CA, USA, pp. 51-61.

Richards, P. (1956). Shock waves on highways, Operations Research 4: 42-51.

Rouphail, N., Tarko, A. and Li, J. (1999). Chapter 9: Traffic flow at signalized intersections, in N. Gartner, C. Messer and A. Rathi (eds), Monograph on Traffic Flow Theory, Oak Ridge National Laboratory, Federal Highway Administration.

Rubio-Ardanaz, J., Wu, J. and Florian, M. (2001). A numerical analytical model for the continuous dynamic network equilibrium problem with limited capacity and spill back, Proceedings of the 2001 IEEE Intelligent Transportation Systems Conference, Oakland, USA.

Sobolev, V. (2001). Brouwer theorem, in M. Hazewinkel (ed.), Encyclopedia of Mathematics, Kluwer.

Sun, X., Munoz, L. and Horowitz, R. (2003). Highway traffic state estimation using improved mixture Kalman filters for effective ramp metering control, Proceedings of the 42th IEEE Conference on Decision and Control, Maui, Hawaii, USA, pp. 6333-6338.

Tampere, C., Corthout, R., Cattrysse, D. and Immers, L. (forthcoming). A generic class of first order node models for dynamic macroscopic simulations of traffic flows, Transportation Research Part B $\mathbf{x x}(\mathrm{xx})$ : xx-xx.

Tampere, C. and Immers, L. (2007). An extended Kalman filter application for traffic state estimation using CTM with implicit mode switching and dynamic parameters, Proceedings of the 10th IEEE Intelligent Transportation Systems Conference, Seattle, USA, pp. 209-216. 
Troutbeck, R. and Brilon, W. (1999). Chapter 8: Unsignalized intersection theory, in N. Gartner, C. Messer and A. Rathi (eds), Monograph on Traffic Flow Theory, Oak Ridge National Laboratory, Federal Highway Administration.

Troutbeck, R. and Kako, S. (1999). Limited priority merge at unsignalized intersections, Transportation Research Part A 33(3-4): 291-304.

TSS Transport Simulation Systems (2006). AIMSUN 5.1 Microsimulator User's Manual Version 5.1.4.

van Hinsbergen, C., Tampere, C., van Lint, J. and van Zuylen, H. (2009). Urban intersections in first order models with the Godunov scheme, Proceedings of mobil.TUM 2009 - International Scientific Conference on Mobility and Transport-ITS for large Cities, Munich, Germany.

van Hinsbergen, C., Zuurbier, F., van Lint, H. and van Zuylen, H. (2008). Macroscopic modelling of intersection delay with linearly decreasing turn capacities, Proceedings of the International Symposium on Dynamic Traffic Assignment, Leuven, Belgium.

Yperman, I., Tampere, C. and Immers, B. (2007). A kinematic wave dynamic network loading model including intersection delays, Proceedings of the 86. Annual Meeting of the Transportation Research Board, Washington, DC, USA.

Zhang, H. and Lin, W. (2001). Some recent developments in traffic flow theory, Proceedings of the IEEE Intelligent Transportation Systems Conference, Oakland (CA), USA, pp. 548-553.

Ziliaskopoulos, A. and Lee, S. (1997). A cell transmission based assignment simulation model for integrated freeway/surface street systems, Proceedings of the 76. Annual Meeting of the Transportation Research Board, Washington, DC, USA. 